This is the author's final, peer-reviewed manuscript as accepted for publication. The publisher-formatted version may be available through the publisher's web site or your institution's library.

\title{
Effects of interband transitions on Faraday rotation in metallic nanoparticles
}

G. M. Wysin, Viktor Chikan, Nathan Young, and Raj Kumar Dani

\section{How to cite this manuscript}

If you make reference to this version of the manuscript, use the following information:

Wysin, G. M., Chikan, V., Young, N., \& Dani, R. K. (2013). Effects of interband transitions on Faraday rotation in metallic nanoparticles. Retrieved from http://krex.ksu.edu

\section{Published Version Information}

Citation: Wysin, G. M., Chikan, V., Young, N., \& Dani, R. K. (2013). Effects of interband transitions on Faraday rotation in metallic nanoparticles. Journal of Physics: Condensed Matter, 25(32), 325302.

Copyright: ( 2013 IOP Publishing Ltd

Digital Object Identifier (DOI): doi:10.1088/0953-8984/25/32/325302

Publisher's Link: http://iopscience.iop.org/0953-8984/25/32/325302/

This item was retrieved from the K-State Research Exchange (K-REx), the institutional repository of Kansas State University. K-REx is available at http://krex.ksu.edu 


\title{
Effects of Interband Transitions on Faraday Rotation in Metallic Nanoparticles
}

\author{
G. M. Wysin, ${ }^{*}$ Viktor Chikan, ${ }^{\dagger}$ Nathan Young, ${ }^{\ddagger}$ and Raj Kumar Dani ${ }^{\S}$ \\ Kansas State University, Manhattan, KS 66506-2601
}

(Dated: May 6, 2013)

\begin{abstract}
The Faraday rotation in metallic nanoparticles is considered based on a quantum model for the dielectric function $\epsilon(\omega)$ in the presence of a DC magnetic field $B$. We focus on effects in $\epsilon(\omega)$ due to interband transitions (IBTs), which are important in the blue and ultraviolet for noble metals used in plasmonics. The dielectric function is found using the perturbation of the electron density matrix due to the optical field of incident electromagnetic radiation. The calculation is applied to transitions between two bands ( $d$ and $p$, for example) separated by a gap, as one finds in gold at the L-point of the Fermi surface. The result of the DC magnetic field is a shift in the effective optical frequency causing IBTs by $\pm \mu_{B} B / \hbar$, where opposite signs are associated with left/right circular polarizations. Faraday rotation for a dilute solution of $17 \mathrm{~nm}$ diameter gold nanoparticles is measured and compared with both the IBT theory and a simpler Drude model for the bound electron response. Effects of the plasmon resonance mode on Faraday rotation in nanoparticles are also discussed.
\end{abstract}

PACS numbers: 77.22.-d, 78.20.Ls, 78.67.-n, 78.67.Bf,

Keywords: quantum Faraday rotation, dielectric function, interband transitions, nanoparticles, plasmons

\section{INTRODUCTION: FARADAY ROTATION ENHANCEMENT AND PLASMON MODES}

There is great interest to design new materials with enhanced Faraday rotation ${ }^{1}$ (FR); these media ${ }^{2,3,4,5}$ are good candidates for applications like field detectors, phase modulators and optical isolators. Nanoparticles (NPs) of radius $a$ much less than the wavelengths $\lambda$ of the electromagnetic (EM) radiation are particularly interesting possibilities, ${ }^{6}$ because one hopes to be able to tune their fundamental physical properties that determine the dielectric permittivity $\epsilon(\omega)$, which influences the FR signal. Further, metallic NPs or NPs with metallic shell coatings have a surface plasmonic mode ${ }^{7}$ where the electron response is greatly enhanced, which leads to increased FR response. ${ }^{8}$ In magnetic core NPs with metallic shell coatings the surface plasmonic mode interacts with a transition in the magnetic core, which is another process that leads to enhanced FR. ${ }^{9}$ Faraday rotation and circular dichroism in NP aggregates of various geometries ${ }^{10}$ offers promise for control of magnetooptical effects. Backscattering of light in disordered media may lead to enhanced Faraday rotation effects. ${ }^{11}$

It is simplest to use a classical Drude term to represent approximately the dielectric response of bound electrons, ${ }^{8}$ or even ignore the bound electron dynamic response, ${ }^{12}$ however, these approaches do not describe the dielectric properties well at higher frequencies. Hui and Stroud ${ }^{13}$ have considered the FR response of a dilute suspension of small particles, with a Drude approximation for NP dielectric function. It is the goal here to compare a classical phenomenological Drude approach with a quantum model appropriate for noble metals such as gold, ${ }^{14,15}$ where interband transitions (IBTs) take place from $d$ to $s p$ bands at the L-point. ${ }^{16}$ We consider a case where the NPs are sufficiently small, so that the primary effect of the DC magnetic field that produces Faraday rotation is a Zeeman splitting of the band states, rather than an entire series of Landau levels. ${ }^{17}$

The surface plasmon frequency $\omega_{s p}$ in a NP in the Rayleigh limit $(a \ll \lambda)$ is considerably less than the bulk plasmon frequency $\omega_{p}$ for the same metal. This is due partly to a geometrical effect, but the more significant reason is that interband transitions taking place above a gap energy greatly modify the dielectric function in the region of the plasmon resonance. For gold, the bulk plasmon, well into the ultraviolet at $138 \mathrm{~nm}$, is moved to around $520-532 \mathrm{~nm}$ for the surface plasmons of NPs. ${ }^{8}$ But to get the correct description, the interband transitions must be taken into account for describing $\epsilon(\omega)$. Here, we include IBTs for the bound electron contribution to $\epsilon(\omega)$, in the presence of a DC magnetic field, so that the Faraday rotation properties can be described.

Faraday rotation is a magneto-optical phenomenon ${ }^{1}$ that measures the fundamental electronic, optical and magnetic response of a dielectric medium. It is similar to optical rotation ${ }^{18}$ except that $\mathrm{FR}$ requires an applied magnetic field. The Faraday rotation is the change in the polarization of an EM wave as it propagates through some medium parallel to the axis of a quasi-static magnetic field. The interaction between the DC field and the charges in the medium leads to different speeds of propagation and different wave vectors $k_{R}$ and $k_{L}$ for the right and left circular polarization components of EM waves, leading to the net rotation of initially linearly polarized waves.

The basic parameter to describe the degree of FR is the Verdet factor, $v$, which is the rotation angle of the polarization per unit propagation length $z$ per applied magnetic field $B$ :

$$
v=\frac{\varphi}{B z}
$$


We consider a composite medium of gold NPs in water. At small volume fraction of gold, $f_{s} \ll 1$, the Verdet factor is linear in the volume fraction. Generally, the rotation $\varphi$ is also linear in magnetic field at low enough fields (although materials of other symmetry ${ }^{19}$ can exhibit quadratic dependence on $B$ ). Then $v$ does not depend on $B$, and we consider only this regime. For dilute composites, the Verdet factor per volume fraction is a better quantity for consideration, defined as

$$
\Upsilon \equiv \frac{v}{f_{s}}=\frac{\varphi}{B z f_{s}}
$$

The Faraday rotation results from the phase difference of the polarizations,

$$
\varphi=\frac{1}{2} \operatorname{Re}\left\{k_{R}-k_{L}\right\} z,
$$

where the respective wave vectors for propagation of the two circular polarizations are determined by relative dielectric functions $\epsilon_{R}$ and $\epsilon_{L}$,

$$
k_{R, L}=\frac{\omega}{c} \sqrt{\mu \epsilon_{R, L}} .
$$

with $\mu$ being the relative magnetic permeability of the medium and $c$ being the speed of light in vacuum. The theoretical description of the FR signal in some metallic or metallic-shell NPs, as a result, requires an accurate description of the dielectric function $\epsilon(\omega)$ for the metal, including the presence of the DC magnetic field. Especially, it is important to have a reliable description of $\epsilon(\omega)$ at shorter wavelengths, into the ultraviolet, below the plasmon frequency for the NPs. Indeed, in order for the theory to correctly predict the plasmon frequency $\omega_{s p}$, requires knowing $\epsilon(\omega)$ all the way into the ultraviolet. Thus, the goal here is to get an accurate theory for $\epsilon(\omega)$ with the DC magnetic field present, that includes interband transitions for the bound electrons as well as the usual plasmon response of the free electron gas.

Below we begin by describing the NP synthesis and a description of the experimental measurement of the Faraday rotation. Then we will continue by summarizing the basic relations among the dielectric functions $\epsilon(\omega)$, $\epsilon_{L}(\omega)$, and $\epsilon_{R}(\omega)$ and the FR response. (All $\epsilon$ are understood to be relative dielectric functions, leading to index of refraction, $n=\sqrt{\mu \epsilon}$.) Next, a simple classical model for $\epsilon(\omega)$ using bound electrons and based on the Drude model is described briefly, for comparison with the quantum calculation for the IBTs. In that model the effect of IBTs is approximated by the response function of a set of bound electrons, with some binding frequency $\omega_{0}$. Part of the motivation for the quantum calculation of the IBTs is to determine the validity of this simpler classical model.

For the quantum effects of IBTs, we adopt the approach used by Boswarva et al. ${ }^{20}$ and also by Adler ${ }^{21}$ of finding the perturbations of the electron density matrix that are caused by only the electric field of the EM waves. The optical magnetic field is ignored. However, the DC magnetic field enters because it shifts the band states. This is a simple Zeeman shift; for nanometersized systems there is no sense to Landau levels that were used by Boswarva et al., and also in theory developed by Halpern et al., ${ }^{22}$ due to the geometric confinement (the NP radii are smaller than the Landau radius $r_{0}=\sqrt{\frac{2 \hbar}{e B}}$, for applied magnetic induction $B$ ). Following through the calculation, there is a contribution to $\epsilon(\omega)$ that requires summing over IBTs with a range of energies. Those integrals are evaluated in two different models: A three-dimensional (3D) band model and a 1D band model. The results are presented both with the presence of a phenomenological electron damping constant $\gamma$ and in a limit that this damping goes to zero. The results are also compared with earlier calculations of the IBT contribution by Inouye et al. ${ }^{14}$ and by Scaffardi and Tocho, ${ }^{15}$ that did not include the DC magnetic field.

The net $\epsilon(\omega)$ includes contributions from both the bound (IBTs) and free (electron gas) electrons. We apply the results to calculate the scaled Verdet factor $\Upsilon$ for a dilute solution of solid metallic NPs, using the parameters for gold in water. The effects of the dilution are considered most simply by the Maxwell-Garnett theory, ${ }^{23,24}$ assuming that the NPs do not aggregate. We find that even with the quantum IBTs included for bound electrons, the experimentally measured FR signal is about $10 \times$ stronger than that predicted by the theory.

We will conclude with comments on the applicability of the results for other systems with plasmonic enhancements of dielectric responses.

\section{SYNTHESIS OF GOLD NPS AND FARADAY ROTATION MEASUREMENTS}

The large gold nanoparticles are prepared from the reduction of $\mathrm{HAuCl}_{4}$ solution by sodium citrate solution as described by Turkevich et al. ${ }^{25}$ Briefly, $5 \mathrm{mg}$ of $\mathrm{HAuCl}_{4}$ and $50 \mathrm{mg}$ of sodium citrate are dissolved in $95 \mathrm{ml}$ and 5 $\mathrm{ml}$ of doubly distilled water, respectively. The $\mathrm{HAuCl}_{4}$ solution is heated to about $70^{\circ} \mathrm{C}$ and the sodium citrate solution is added, vigorously stirring the solution for 50 minutes. The color of the solution gradually changes from faint pink to wine red. The resulting large gold nanoparticles have size $17 \pm 3 \mathrm{~nm}$. Assuming 100\% reduction of the gold into NPs, the upper limit of volume fraction of gold in the solution is $f_{s}=1.50 \times 10^{-6}$. By analyzing the extinction coefficient of the solution by the techniques in Ref. 26, the actual volume fraction is estimated to be $f_{s}=1.23 \times 10^{-6}$.

The Faraday rotation spectrum of NPs in water solution was measured with the help of a home built pulsed magnet. The magnet consists of a helical coil machined from a copper beryllium block and electroplated with 
silver. The pulsed current to the coil is provided via a simple RLC circuit. The capacitor bank of $77.3 \mu \mathrm{F}$ from Maxwell Laboratories is charged by a power supply/charger of Lumina Power, Inc. The power supply uses $100-240 \mathrm{~V}$ AC-50/60 Hz input and output of $10 \mathrm{kV}$ at $500 \mathrm{~J} / \mathrm{s}$ in continuous operation. The charge from the capacitor bank is discharged into the coil via a high voltage trigger spark gap. The current is monitored in the circuit via a Rogowski coil, which measures the current derivative.

The Faraday rotation of the nanoparticle solutions is measured in a plastic cell placed in the coil. A flash light source is triggered along with the pulsed magnet that allows the synchronization of the magnet with the optical measurement. The duration of the light pulse is $1.4 \mu \mathrm{s}$, while the duration of the magnetic pulse is $\sim 50 \mu \mathrm{s}$, which allows that during the optical measurement the magnetic field is relatively constant. In front of the flash light source a polarizer is placed to produce polarized light for the Faraday measurement. The polarized light passes through the sample containing the nanoparticle solutions. The light leaving the optical cell passes through another polarizer that is set to 45 degree with respect the first polarizer. The light then enters a fiber optic spectrometer, which is also synchronized with the pulsed magnet and the light source. The Faraday rotation is calculated from the intensity change in the spectrum before and after the magnetic pulse. The magnetic field and the Faraday rotation setup are calibrated with water placed into the optical cell. The measurements are taken at 4.2 tesla magnetic fields.

\section{THEORY: DIELECTRIC POLARIZATION, CURRENTS, $\epsilon(\omega)$ AND FARADAY ROTATION}

We consider EM radiation at frequency $\omega$ with the electric field $\mathbf{E}(t) \sim e^{-i \omega t}$, incident on a material particle (an individual NP) much smaller then the wavelength (the Rayleigh limit). Then the field $\mathbf{E}$ is taken as uniform inside the sample. The dielectric properties are based on the averaged dipole moment of the electrons of charge $e$, $\mathbf{d}=e \mathbf{r}$. For $n=N / V$ electrons per unit volume, the electric polarization can be expressed as

$$
\mathbf{P}=n\langle\mathbf{d}\rangle=\tilde{\chi} \cdot \epsilon_{0} \mathbf{E}
$$

where $\epsilon_{0}$ is the permittivity of vacuum and $\tilde{\chi}$ is the susceptibility tensor that is to be found. The dielectric function considered as a tensor $\tilde{\epsilon}$ is defined via the electric displacement $\mathbf{D}=\epsilon_{0} \tilde{\epsilon} \cdot \mathbf{E}$ or

$$
\mathbf{D}=\epsilon_{0} \mathbf{E}+\mathbf{P},
$$

from which the usual definition results,

$$
\tilde{\epsilon}=\mathbf{1}+\tilde{\chi}
$$

It is useful to realize another way to get to $\tilde{\epsilon}$, via averaging of the microscopic currents, i.e., those caused by the optical fields. The dielectric medium under study has current density $\mathbf{J}$, which combines with the vacuum displacement current. In this view the Ampere/Maxwell Law is

$$
\nabla \times \mathbf{H}=\mathbf{J}+\epsilon_{0} \frac{\partial \mathbf{E}}{\partial t} .
$$

All the effects of the medium are contained in $\mathbf{J}$. This must be equivalent to the alternative viewpoint that the currents are represented instead by a dielectric function,

$$
\nabla \times \mathbf{H}=\frac{\partial \mathbf{D}}{\partial t} .
$$

Considered at the frequency of the EM radiation with time derivatives $\partial / \partial t \rightarrow-i \omega$, these alternate views give

$$
\mathbf{J}=-i \omega \epsilon_{0}(\tilde{\epsilon}-\mathbf{1}) \cdot \mathbf{E}=-i \omega \tilde{\chi} \cdot \epsilon_{0} \mathbf{E}
$$

Thus, an averaging of the microscopic currents will also lead to the susceptibility and dielectric tensors.

We assume that the DC magnetic field $\mathbf{B}$ is along the $\hat{z}$-direction, the same as the propagation direction of the EM waves, with wave vector $\mathbf{k}=k \hat{z}$. Then the electric field in the waves has only $x y$ components; only the transverse part of the dielectric tensor is needed. In this situation it has the following symmetry ${ }^{27}$

$$
\tilde{\epsilon}=\left[\begin{array}{cc}
\epsilon_{x x} & \epsilon_{x y} \\
-\epsilon_{x y} & \epsilon_{x x}
\end{array}\right]=\left[\begin{array}{cc}
\epsilon_{x x} & i \mathcal{E}_{x y} \\
-i \mathcal{E}_{x y} & \epsilon_{x x}
\end{array}\right]
$$

The off-diagonal elements are determined by the DC magnetic field; they vanish when $\mathbf{B}=0$. The variable $\mathcal{E}_{x y}=-i \epsilon_{x y}$ is convenient later; it is real in the absence of electron damping. The EM waves that propagate without any change in polarization are those with polarization vectors that are eigenvectors of $\tilde{\epsilon}$. These eigenstates are the usual states of right and left circular polarization. Thus, solving the eigenvector problem, $\tilde{\epsilon} \cdot \hat{\mathbf{u}}_{i}=\epsilon_{i} \hat{\mathbf{u}}_{i}, \quad i=1,2$, with eigenvalues $\epsilon_{i}$ and eigenvectors $\hat{\mathbf{u}}_{i}$, one finds the right circular polarization state (negative helicity) with $E_{y}=-i E_{x}$ :

$$
\epsilon_{R}=\epsilon_{x x}+\mathcal{E}_{x y}, \quad \hat{\mathbf{u}}_{R}=\frac{1}{\sqrt{2}}(\hat{x}-i \hat{y}),
$$

and the left circular polarization state (positive helicity) with $E_{y}=+i E_{x}$ :

$$
\epsilon_{L}=\epsilon_{x x}-\mathcal{E}_{x y}, \quad \hat{\mathbf{u}}_{L}=\frac{1}{\sqrt{2}}(\hat{x}+i \hat{y}) .
$$

Each mode has a different wave vector for propagation, according to expression (4). Then starting from a linearly polarized wave at position $z=0$, its right and left circular components get out of phase by the time it 
travels to position $z$, leading to the rotation of the polarization through the angle $\varphi$ given in expression (3). One might also mention, that in general, the dielectric tensor elements are complex, then there is also a change in ellipticity $\mathcal{X}$ of the polarization, given from the imaginary part,

$$
\mathcal{X}=\frac{1}{2} \operatorname{Im}\left(k_{R}-k_{L}\right) z .
$$

The two effects of Faraday rotation and change in ellipticity $(\tan \mathcal{X}=$ ratio of minor to major axis of the ellipse swept out by the electric vector) can be combined into one complex parameter, ${ }^{28}$

$$
\psi=\varphi+i \mathcal{X}=\frac{1}{2}\left(k_{R}-k_{L}\right) z
$$

Usually these effects are extremely small and close to linear in B. Then there is only a tiny difference in $k_{R}$ and $k_{L}$, which gives to a very good approximation, the complex relation,

$$
\psi=\varphi+i \mathcal{X} \approx \frac{\omega}{2 c} \sqrt{\frac{\mu}{\epsilon_{x x}}} \mathcal{E}_{x y} z
$$

This emphasizes how the components of $\tilde{\epsilon}$ are needed to describe the changes in the optical polarization.

From the experimental perspective, the measurement of the absorption (or, attenuation) coefficient $\alpha$ is at least one technique that sets a relative scale for the FR. It is given from

$$
\alpha=2 \operatorname{Im}\left\{k_{\mathrm{eff}}\right\}=2 \frac{\omega}{c} \operatorname{Im}\left\{\sqrt{\mu \epsilon_{\mathrm{eff}}}\right\} .
$$

This could use either $\epsilon_{R}$ or $\epsilon_{L}$ or their average for the effective dielectric function $\epsilon_{\text {eff }}$ of the medium, as this expression does not involve their difference, which is extremely small. Thus, measurements of $\alpha$ serve to set some unknown fitting parameters, when needed.

\section{CLASSICAL PHENOMENOLOGICAL MODEL FOR $\epsilon(\omega)$ (DRUDE MODEL)}

In this section the electron motion is assumed to be classical. An electron of bare mass $m_{0}$ and charge $e=$ $-1.602 \times 10^{-19} \mathrm{C}$ has some trajectory $\mathbf{r}(t)=(x(t), y(t))$ in response to all forces acting on it, and the averaging of its induced electric dipole moment $\mathbf{d}=e \mathbf{r}$ lead to the dielectric function.

To include the effect of the constant $\mathbf{B}$ on $\tilde{\epsilon}$ it is assumed that there are two primary contributions to the dielectric response. The first is the contribution of free electrons with number density $n$, and some damping parameter $\gamma_{p}$, that leads to the usual plasmon response with a plasma frequency $\omega_{p}^{2}=n e^{2} / m \epsilon_{0}$. The second is a contribution due to bound electrons, with some binding frequency $\omega_{0}$ and another damping parameter $\gamma_{0}$. The contribution of bound electrons is essential to describe $\epsilon(\omega)$ correctly $^{15}$ in NPs.

Any electron, whether free or bound, is acted on as well by the electric force from the optical field, and the Lorentz force from the DC magnetic field. The force due to the optical magnetic field can be ignored in lowest order. In this Drude approximation the equation of motion of a bound electron is ${ }^{29}$

$$
m_{o} \ddot{\mathbf{r}}=e \mathbf{E}+e \dot{\mathbf{r}} \times \mathbf{B}-m_{o} \omega_{0}^{2} \mathbf{r}-m_{o} \gamma_{0} \dot{\mathbf{r}}
$$

Under the assumption of $e^{-i \omega t}$ time dependence of the optical field E, which is the source field, this is

$$
\left[m_{o}\left(\omega_{0}^{2}-\omega^{2}-i \omega \gamma_{0}\right)-i \omega e \mathbf{B} \times\right] \mathbf{r}=e \mathbf{E}
$$

In terms of the components this is a matrix relation,

$$
\left[\begin{array}{cc}
\omega_{0}^{2}-\omega^{2}-i \omega \gamma_{0} & +i \omega \omega_{B} \\
-i \omega \omega_{B} & \omega_{0}^{2}-\omega^{2}-i \omega \gamma_{0}
\end{array}\right]\left[\begin{array}{l}
x \\
y
\end{array}\right]=\frac{e}{m_{o}}\left[\begin{array}{c}
E_{x} \\
E_{y}
\end{array}\right],
$$

where the cyclotron frequency with $\mathbf{B}$ along $\hat{z}$ is

$$
\omega_{B}=\frac{e B}{m_{o}} .
$$

The matrix $\tilde{\Omega}^{2}$ on the LHS of (20) has the same kind of symmetry as that of $\tilde{\epsilon}$ in (11), because the diagonal elements are equal and the imaginary off-diagonal elements differ only in sign. This means $\tilde{\Omega}^{2}$ has the same eigenvectors, which are the right and left circular polarization states. Based on its structure, the eigenvalues $\Omega_{R}^{2}$ and $\Omega_{L}^{2}$ of $\tilde{\Omega}^{2}$ are easy to read out. For right circular polarization,

$$
\Omega_{R}^{2}=\omega_{0}^{2}-\omega^{2}-i \omega \gamma_{0}+\omega \omega_{B} .
$$

For left circular polarization, the last term (from the off-diagonal element) has the opposite sign,

$$
\Omega_{L}^{2}=\omega_{0}^{2}-\omega^{2}-i \omega \gamma_{0}-\omega \omega_{B} .
$$

The effect of the DC magnetic field appears only in the last factor. These two eigenvalues can be combined into a single convenient expression in terms of the helicity $\nu=-1$ for right circular polarization and $\nu=+1$ for left circular polarization:

$$
\Omega_{\nu}^{2}=\omega_{0}^{2}-\omega^{2}-i \omega \gamma_{0}-\nu \omega \omega_{B}
$$

The helicity is the projection of the photon intrinsic angular momentum $\vec{L}$ on the direction of propagation (k or $\hat{z}$ ). In this expression it multiplies the magnetic field component along the same axis. Any physical differences for right and left circular polarizations will become interchanged if the direction of the magnetic field is reversed. In terms of a vector $\vec{\nu}=\vec{L} / \hbar$, the last factor in these eigenvalues could be written most generally as $\omega \vec{\nu} \cdot \vec{\omega}_{B}$. 
If the electric field contains only one of the circular polarizations, i.e., $\mathbf{E}=E_{\nu} \hat{\mathbf{u}}_{\nu}$, the response $\mathbf{r}$ also will be proportional to the same eigenvector. Then the solution for the electron position is very simple,

$$
\mathbf{r}=\frac{e E_{\nu}}{m_{o} \Omega_{\nu}^{2}} \hat{\mathbf{u}}_{\nu} .
$$

The factor $e / m \Omega_{\nu}^{2}$ gives the size of the response for this polarization. Here we see the fundamental physical difference between the polarizations. One of the polarizations causes a larger circular motion of the electrons than the other polarization. Which one actually is larger depends on the relation between $\omega_{0}$ and $\omega$. This difference leads to a corresponding difference in the dielectric effects.

Based on this position response, it is then easy to find the effective dielectric functions for the two circular polarizations, using $\mathrm{d}=e \mathrm{r}$. The susceptibility due to these electrons is $\chi=n\langle\mathrm{~d}\rangle / \epsilon_{0} E$. The result can be summarized in a single formula,

$$
\chi_{\nu}(\omega)=\frac{n e^{2}}{m_{o} \epsilon_{0} \Omega_{\nu}^{2}},
$$

where $\nu=-1 /+1$ refers to $R / L$ polarizations, respectively. This applies to separately, the contribution from the bound electrons, or, the contribution of the free electrons, using appropriate parameters in each case.

Look at this another way. An arbitrary electric field can be expressed either as $\mathbf{E}=E_{x} \hat{x}+E_{y} \hat{y}$ or as $\mathbf{E}=$ $E_{R} \hat{\mathbf{u}}_{R}+E_{L} \hat{\mathbf{u}}_{L}$, where

$$
\begin{array}{ll}
E_{R}=\frac{1}{\sqrt{2}}\left(E_{x}+i E_{y}\right), & E_{x}=\frac{1}{\sqrt{2}}\left(E_{R}+E_{L}\right), \\
E_{L}=\frac{1}{\sqrt{2}}\left(E_{x}-i E_{y}\right), & E_{y}=\frac{-i}{\sqrt{2}}\left(E_{R}-E_{L}\right) .
\end{array}
$$

One can combine the right and left solutions and get the general solution for any electric field, in diagonal form:

$$
\mathbf{r}=\frac{e E_{R}}{m_{o} \Omega_{R}^{2}} \hat{\mathbf{u}}_{R}+\frac{e E_{L}}{m_{o} \Omega_{L}^{2}} \hat{\mathbf{u}}_{L}
$$

Alternatively, this can be written in Cartesian components,

$$
\begin{aligned}
& x=\frac{1}{\sqrt{2}} \frac{e}{m_{o}}\left[\frac{E_{R}}{\Omega_{R}^{2}}+\frac{E_{L}}{\Omega_{L}^{2}}\right], \\
& y=\frac{1}{\sqrt{2}} \frac{i e}{m_{o}}\left[\frac{E_{R}}{\Omega_{R}^{2}}-\frac{E_{L}}{\Omega_{L}^{2}}\right] .
\end{aligned}
$$

Simplifying, or inverting the matrix equation (20), leads to the general electron motion,

$$
\left[\begin{array}{l}
x \\
y
\end{array}\right]=\frac{e / m_{o}}{\Omega_{R}^{2} \Omega_{L}^{2}}\left[\begin{array}{cc}
\omega_{0}^{2}-\omega^{2}-i \omega \gamma_{0} & -i \omega \omega_{B} \\
+i \omega \omega_{B} & \omega_{0}^{2}-\omega^{2}-i \omega \gamma_{0}
\end{array}\right]\left[\begin{array}{c}
E_{x} \\
E_{y}
\end{array}\right]
$$

Multiplied by $e$, the relation shows the polarizability matrix of the electron. This expression leads to the susceptibility tensor,

$$
\tilde{\chi}=\frac{n e^{2} / m_{o} \epsilon_{0}}{\Omega_{L}^{2} \Omega_{R}^{2}}\left[\begin{array}{cc}
\omega_{0}^{2}-\omega^{2}-i \omega \gamma_{0} & -i \omega \omega_{B} \\
+i \omega \omega_{B} & \omega_{0}^{2}-\omega^{2}+i \omega \gamma_{0}
\end{array}\right] .
$$

One can see this is consistent with (26), because its eigenvalues are $\chi_{R}=\chi_{x x}-i \chi_{x y}$ and $\chi_{L}=\chi_{x x}+i \chi_{x y}$, which agrees exactly with (26).

\section{A. Combination of free and bound electron responses}

Now to use this to describe a metal such as gold, we assume first there is some density of free electrons $n$, with a bulk plasma frequency $\omega_{p}^{2}=n e^{2} / m_{o} \epsilon_{0}$, a damping $\gamma_{p}$ and a zero binding frequency. In addition, there is some other density $n_{0}$ of bound electrons, leading to an effective weight $g_{0}^{2}=n_{0} e^{2} / m_{o} \epsilon_{0}$, with an associated damping $\gamma_{0}$ and binding frequency $\omega_{0}$. The net dielectric function is the sum of the two contributions to $\tilde{\chi}$. In terms of the polarization states $\nu= \pm 1$, the dielectric function is taken as

$\epsilon_{\nu}(\omega)=1-\frac{\omega_{p}^{2}}{\omega^{2}+i \omega \gamma_{p}+\nu \omega \omega_{B}}-\frac{g_{0}^{2}}{\omega^{2}-\omega_{0}^{2}+i \omega \gamma_{0}+\nu \omega \omega_{B}}$.

The first two terms are the usual ones for describing a free electron gas. The last term uses the single resonance to approximate the effects of bound electrons. Both include the DC magnetic field implicitly in the cyclotron frequency, $\omega_{B}$. The ease with which the magnetic field is included in the bound electron response is the main advantage of this model.

One can produce the Cartesian elements of $\tilde{\epsilon}$, for instance, using (12) and (13), by the combinations of these eigenvalues:

$$
\begin{aligned}
& \epsilon_{x x}=\epsilon_{y y}=\frac{1}{2}\left(\epsilon_{R}+\epsilon_{L}\right), \\
& \epsilon_{x y}=-\epsilon_{y x}=i \mathcal{E}_{x y}=\frac{i}{2}\left(\epsilon_{R}-\epsilon_{L}\right) .
\end{aligned}
$$

\section{B. Maxwell-Garnett averaging for dilute solutions}

The medium of interest here is actually a dilute solution of NPs at a volume fraction $f_{s} \ll 1$ in a host liquid, which we take as water, with its host dielectric constant $\epsilon_{h}=1.777$. The NPs are considered the scatterers with dielectric function $\epsilon_{s}$ For comparison with experiment, the effective dielectric function $\epsilon_{\text {eff }}$ of the solution is required. The theory for calculating the effective dielectric function depends somewhat on the assumption of how the particles are dispersed in the liquid. In the simplest approximation, they are assumed 
to be randomly dispersed and not sticking to each other. In the Maxwell-Garnett (MG) theory, ${ }^{23,24}$ one finds the volume-averaged electric field and the volume-averaged polarization response to that field, from which $\epsilon_{\text {eff }}$ is determined. The MG theory is known to apply well even in the presence of multiple-scattering. ${ }^{30}$ These volume averages are

$$
\begin{aligned}
& \mathbf{E}_{\mathrm{av}}=f_{s} \mathbf{E}_{s}+\left(1-f_{s}\right) \mathbf{E}_{h}, \\
& \mathbf{P}_{\mathrm{av}}=f_{s} \mathbf{P}_{s}+\left(1-f_{s}\right) \mathrm{P}_{h},
\end{aligned}
$$

where $s$ and $h$ refer to the values in the scatterer and the host, respectively. For spherical scatterers exposed to asymptotic field $\mathbf{E}_{h}$ in the host, the Clausius-Mosotti equation gives the internal fields,

$$
\mathbf{E}_{s}=\frac{3 \epsilon_{h}}{\epsilon_{s}+2 \epsilon_{h}} \mathbf{E}_{h}, \quad \mathbf{P}_{s}=\left(\epsilon_{s}-1\right) \epsilon_{0} \mathbf{E}_{s}
$$

Then with polarization $\mathbf{P}_{h}=\left(\epsilon_{h}-1\right) \epsilon_{0} \mathbf{E}_{h}$ in the host, one finds the average

$$
\epsilon_{\mathrm{eff}}=1+\frac{\mathbf{P}_{\mathrm{av}}}{\epsilon_{0} \mathbf{E}_{\mathrm{av}}}=\epsilon_{h} \frac{1+2 \beta_{f}}{1-\beta_{f}},
$$

which involves the scaled volume fraction $\left(f_{s}\right.$ is the fraction of volume occupied by NPs in the solution),

$$
\beta_{f}=f_{s} \frac{\epsilon_{s}-\epsilon_{h}}{\epsilon_{s}+2 \epsilon_{h}} .
$$

This MG averaging procedure for composite systems is usually summarized by the equivalent relation,

$$
\frac{\epsilon_{\mathrm{eff}}-\epsilon_{h}}{\epsilon_{\mathrm{eff}}+2 \epsilon_{h}}=f_{s} \frac{\epsilon_{s}-\epsilon_{h}}{\epsilon_{s}+2 \epsilon_{h}}
$$

Expression (40) can be applied separately to the left and right circular polarization states, then leading to an effective dielectric function for each, that will then give the Faraday rotation (3) for a dilute solution.

\section{Classical model parameters for gold nanoparticles}

Based on the work in Ref. 8, the parameters needed for this classical model were found by fitting it to the absorption measured experimentally with $B=0$, for a dilute solution of $17 \mathrm{~nm}$ diameter gold NPs in water. That fitting is based on using the effective dielectric function $\epsilon_{\text {eff }}$ from the MG theory, to give the absorption in the solution, according to expression (17).

For this classical Drude model, based on the electron number density, and using effective mass equal to the bare electron mass, the bulk plasma frequency is $\omega_{p}=1.37 \times 10^{16} \mathrm{rad} / \mathrm{s}$, which corresponds to $\lambda_{p}=$ $2 \pi c / \omega_{p}=138.5 \mathrm{~nm}$. The damping of the free electrons in

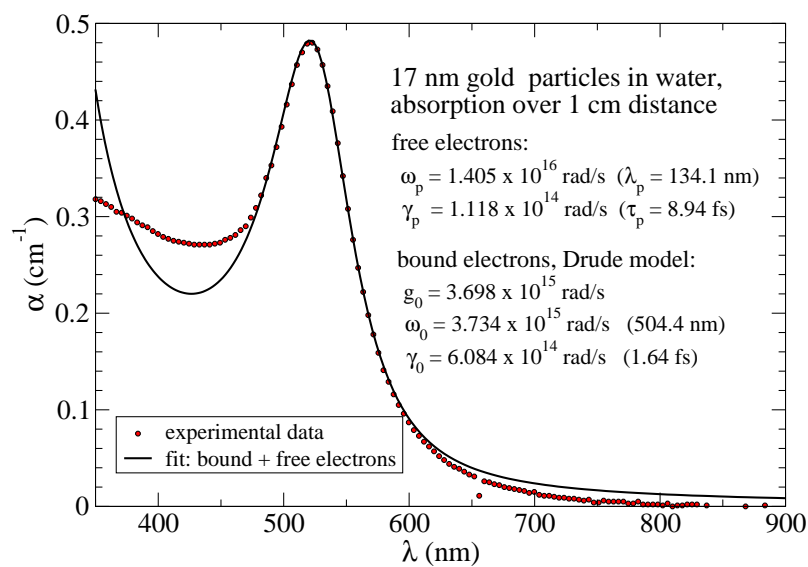

FIG. 1: (Color online) Fitting of the absorption of $17 \mathrm{~nm}$ diameter gold particles in water solution, according to the Drude model for the bound electrons. Parameters indicated are used to get a good fit to the absorption peak near 522 $\mathrm{nm}$. The fitted volume fraction of gold is $f_{s}=3.36 \times 10^{-6}$.

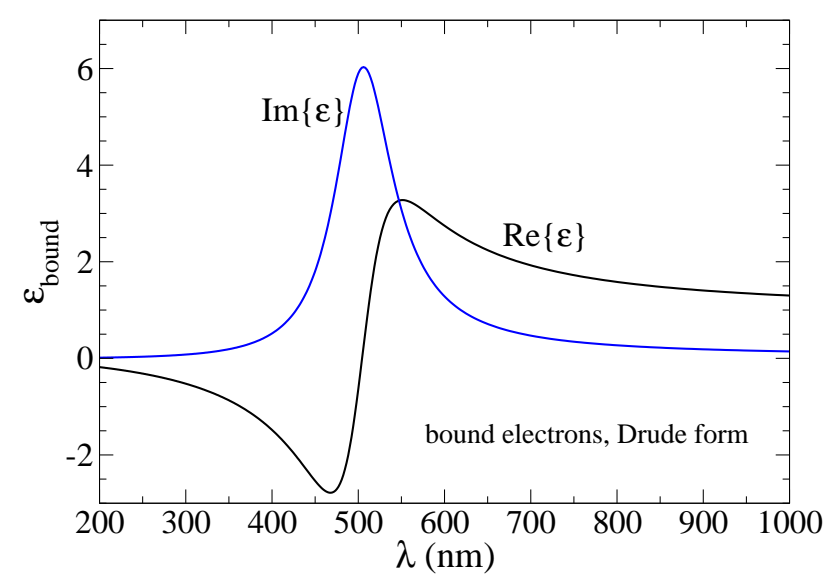

FIG. 2: (Color online) The bound electron contribution to the permittivity, from the second term of Eq. (34), according to the Drude model for the bound electrons, using the parameters of Figure 1. The real part of $\epsilon(\omega)$ becomes negative for frequencies above $\omega_{0}$ (wavelength $504 \mathrm{~nm}$ ), which is a defect of this model.

NPs can have an intrinsic term and a surface scattering term. Thus a size-dependent damping factor is included, according to the combination of these processes, ${ }^{31}$

$$
\gamma_{p}=\frac{1}{\tau}+\frac{v_{F}}{d}
$$

where $\tau \approx 9.1$ fs is the intrinsic scattering time, $v_{F}=$ $1.40 \times 10^{6} \mathrm{~m} / \mathrm{s}$ is the Fermi velocity, and $d$ is the thickness of the gold. This thickness could be the diameter for solid spherical particles, or, the thickness of a shell for core/shell particles. We discuss data for gold particles of 


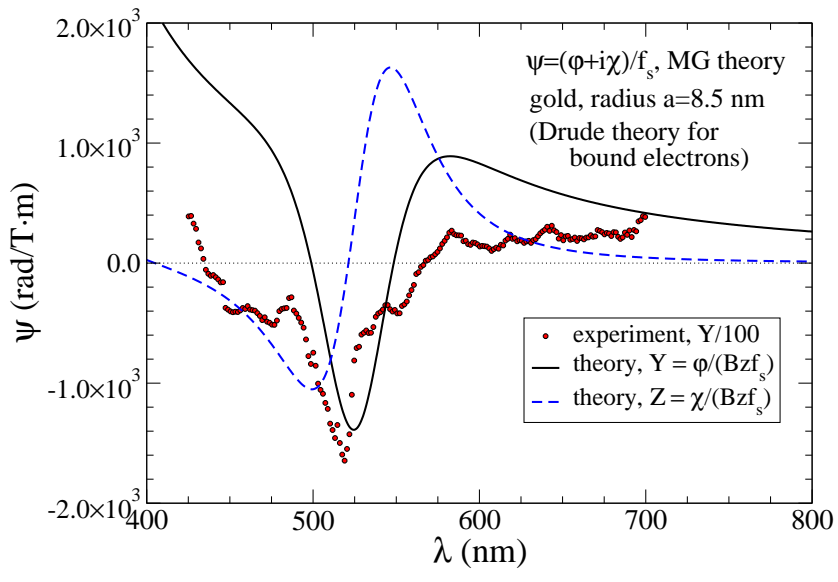

FIG. 3: (Color online) Faraday rotation for $17 \mathrm{~nm}$ diameter gold NPs, from experiment, and according to the Drude model for the bound electrons, using the Drude fitting parameters of Figure 1. The experimental results have been scaled by $1 / 100$ to allow them to be plotted together with the Drude theory. The theory result was obtained with the MG effective medium approach. Faraday rotation angle $\varphi$ and ellipticity angle $\mathcal{X}$ have been scaled by $B, z, f_{s}$, to give $\Psi=\Upsilon+i Z$.

average diameter $17 \mathrm{~nm}$; the prediction for their effective damping is then $\gamma_{p}=1.92 \times 10^{14} \mathrm{rad} / \mathrm{s}$, or, a time scale $\tau_{p}=\gamma_{p}^{-1}=5.20 \mathrm{fs}$.

The Drude theory was fitted to experimental data for absorption through a $1 \mathrm{~cm}$ path of water solution of gold particles with average diameter of $17 \mathrm{~nm}$. The fitting parameters were chosen to get a good description of the absorption peak present near $522 \mathrm{~nm}$, attributed to surface plasmon response. A good description can be obtained while also allowing the volume fraction and free electron parameters $\omega_{p}$ and $\gamma_{p}$ to vary, see Figure 1. The contribution from the bound electrons can be represented approximately using the amplitude parameter $g_{0}=3.70 \times 10^{15} \mathrm{rad} / \mathrm{s}$, the binding frequency $\omega_{0}=3.73 \times 10^{15} \mathrm{rad} / \mathrm{s}$ (wavelength $504 \mathrm{~nm}$ ), and damping frequency $\gamma_{0}=6.08 \times 10^{15} \mathrm{rad} / \mathrm{s}$, which corresponds to a damping time of $\tau_{0}=1 / \gamma_{0} \approx 1.64 \mathrm{fs}$. To get this good fit to the peak, the free electrons are at the same time represented using plasma frequency $\omega_{p}=1.40 \times 10^{16} \mathrm{rad} / \mathrm{s}$, equivalent to $\lambda_{p}=134.1 \mathrm{~nm}$, and a damping $\gamma_{p}=1.118 \times 10^{14} \mathrm{rad} / \mathrm{s}$, corresponding to the damping time $\tau_{p}=8.94 \mathrm{fs}$. These are slightly different than the accepted bulk values, however, we consider them here only as a model that fits accurately the absorption peak.

From these fitted dielectric parameters, the theoretical Faraday rotation response can be obtained. Results for the Faraday rotation and ellipticity for $17 \mathrm{~nm}$ gold NPs in solution are shown in Fig. 3. The complex rotation angle $\psi$ is found from Eq. 15, together with applying the
Maxwell-Garnett procedure for the composite medium, Eq. 40, for the effective dielectric function of the composite solution. We have scaled the rotation angle $\varphi$ and ellipticity $\mathcal{X}$ by the product of path length $z$, magnetic field $B$ and gold volume fraction $f_{s}$, to remove the linear dependence on these quantities. Thus we define the complex rotation angle scaled by volume fraction,

$$
\Psi \equiv \Upsilon+i Z=\psi / f_{s}
$$

Then, $\Upsilon \equiv \varphi /\left(B z f_{s}\right)$ is the Verdet factor per unit volume fraction, and $Z \equiv \mathcal{X} /\left(B z f_{s}\right)$ is a corresponding ellipticity factor per unit volume fraction. Then the results for $\Upsilon$ and $Z$ do not depend on $B$, $z$, or $f_{s}$ in the linear regime. The experimental data for $\Upsilon$ are also displayed in Fig. 3, scaled down by a factor of 1/100 in order to be shown together with the theory.

One sees that the model predicts a negative peak in the Faraday rotation near $525 \mathrm{~nm}$, apparently associated with the plasmon resonance (see 28 for the distinction between positive and negative rotation angles). The experimental data have a similar negative peak in the same region, although its magnitude is significantly larger than this theory predicts. The theory has a wider positive peak around $580 \mathrm{~nm}$ and a long tail at longer wavelengths, but this positive peak is rather weak in the experimental data. For the ellipticity, the main feature predicted is a positive peak around $540 \mathrm{~nm}$, slightly above the plasmon wavelength, together with its associated negative peak and long tail at shorter wavelengths. Unfortunately, the model exhibits an artifact at shorter wavelengths: both $\varphi$ and $\mathcal{X}$ tend to increase greatly at short wavelengths in an unphysical behavior. This is due to the fact that classical Drude model cannot correctly describe the bound electron response at higher frequencies.

This model is an approximate way to include the effect of $B$ on classical bound electrons, however, it should be replaced by the more complete calculation using the quantum interband transitions presented later. It gives a reasonable fit to the absorption curve from $900 \mathrm{~nm}$ down to $400 \mathrm{~nm}$, however, below that wavelength it predicts much more absorption than actually takes place. Also, this Drude description of the bound electrons cannot accurately describe the response in the wavelengths $350-500 \mathrm{~nm}$. This model does not require any background (i.e., high-frequency) dielectric function $\epsilon_{\infty} \sim 10$, as has been applied in other studies to mimic the effect of bound electrons. ${ }^{31}$ Even so, the fit to the absorption peak due to the SP mode is very good, while the corresponding negative FR peak due to the SP mode of 17 $\mathrm{nm}$ gold particles is about 100 times stronger than the theory predicts. 


\section{QUANTUM DESCRIPTION OF $\epsilon(\omega)$ VIA PERTURBATION OF THE DENSITY MATRIX}

In this Section we consider the quantum calculation of the effects due to bound electrons, which is taken into account by finding contributions to $\epsilon(\omega)$ due to interband transitions, in the presence of the DC magnetic field. The electrons are considered non-interacting.

The single-electron Hamiltonian is taken as

$$
\hat{H}=\frac{1}{2 m_{o}}[\hat{\mathbf{p}}-e \hat{\mathbf{A}}(\hat{\mathbf{r}}, t)]^{2}+e \hat{\phi}(\hat{\mathbf{r}}, t)+\hat{U}(\hat{\mathbf{r}}),
$$

where the charge is $e, \hat{\phi}$ and $\hat{\mathbf{A}}$ are the scalar and vector potentials of the EM fields, and $\hat{U}$ is the periodic potential of the lattice. The canonical momentum operator for the electron is $\hat{\mathbf{p}}=-i \hbar \vec{\nabla}$. The more physical momentum is the kinetic momentum operator,

$$
\vec{\pi}=\hat{\mathbf{p}}-e \hat{\mathbf{A}},
$$

because it is the square of this operator that determines the energy. We take the scalar potential as zero (Coulomb gauge). The vector potential $\hat{\mathbf{A}}$ includes a term for the DC magnetic field, $\hat{\mathbf{A}}_{0}$ and a term for the AC optical field $\hat{\mathbf{A}}_{1}$. The optical field is treated as a classical non-quantized field, that oscillates as $e^{-i \omega t}$.

The electron bands, unperturbed by optical fields, come from the solution of a Hamiltonian with the kinetic energy, the lattice periodic potential, and the DC magnetic field,

$$
\hat{H}_{0}=\frac{1}{2 m_{o}}\left(\hat{\mathbf{p}}-e \hat{\mathbf{A}}_{0}\right)^{2}+U(\mathbf{r}) .
$$

At weak enough DC magnetic field, the quadratic term in $\hat{\mathbf{A}}_{0}$ can be dropped, and the effect of the cross term with $\hat{\mathbf{p}}$ is the orbital Zeeman splitting,

$$
\hat{H}_{0}=\frac{\hat{\mathbf{p}}^{2}}{2 m_{o}}+U(\mathbf{r})-\vec{\mu} \cdot \mathbf{B} .
$$

The magnetic dipole moment due to the orbital angular momentum is

$$
\vec{\mu}=\frac{e}{2 m_{o}} \vec{L} .
$$

As the electron charge is negative, $\vec{\mu}$ points opposite to $\vec{L}$. The component of $\vec{\mu}$ along the magnetic field is $m \mu_{B}$, where $\mu_{B}=e \hbar / 2 m_{o}$ is the (negative) Bohr magneton, and $m=m_{l}$ is the magnetic quantum number. The Zeeman splitting is an energy shift $\Delta E=-m \mu_{B} B=$ $-\frac{1}{2} m \hbar \omega_{B}$, where both $\mu_{B}$ and $\omega_{B}$ can be negative, due to the negative electron charge. The states of this Hamiltonian are some electron band states, including any Zeeman shifts,

$$
\hat{H}_{0}|\mathbf{k} l m\rangle=E_{\mathbf{k} l m}|\mathbf{k} l m\rangle .
$$

The band states, labeled by wave vector $\mathbf{k}$ and angular indexes $l, m$, have wave functions

$$
\psi_{\mathbf{k} l m}(\mathbf{r})=\langle\mathbf{r} \mid \mathbf{k} l m\rangle=\frac{1}{\sqrt{V}} e^{i \mathbf{k} \cdot \mathbf{r}} u_{\mathbf{k} l m}(\mathbf{r})
$$

These can be considered the original states of an unperturbed problem. The optical field is the perturbation on these states, whose effect is studied using the density matrix approach.

Because we consider states in NPs, the DC magnetic field only produces Zeeman shifts, rather than Landau levels. Due to the geometrical confinement, there is no sense to Landau levels that would have extended wave functions much larger than the size of the particles. For instance, at a small applied field strength $B=0.1$ tesla, the length scale of the Landau levels is the Landau radius, $r_{0}=\sqrt{\frac{2 \hbar}{e B}}=115 \mathrm{~nm}$. This is much larger than the radius of the nanoparticles under consideration, typically from $5-10 \mathrm{~nm}$. The Landau wave functions do not fit into the NPs at this field strength, giving a non-bulk situation. The degeneracy of Landau levels is on the order of $\left(R / r_{0}\right)^{2}$, where $R$ is the system radius. At $B=0.1$ tesla, the degeneracy is about $(8.5 / 115)^{2} \approx 0.0055$, however, this fractional value is not meaningful. For the larger magnetic field $B=4.2 \mathrm{~T}$, the Landau radius is reduced to $r_{0}=17.7 \mathrm{~nm}$. This is still somewhat larger that the NP radius of $8.5 \mathrm{~nm}$, and the degeneracy is about $(8.5 / 17.7)^{2} \approx 0.23$, still significantly less than 1 , so the theory should be applicable.

These considerations show that the Landau levels are the incorrect solutions in a confined geometry. When one looks more carefully at how to arrive at the quantum solution, the radial wave functions should go to zero at the boundary of the NP (for bound electrons). For spherical particles, that radial dependence would be described by spherical Bessel functions, $j_{l}(k r)$, with discrete allowed $k$, and angular dependence described by spherical harmonics for a chosen angular momentum, $l, m$. We consider a quasi-bulk approximation, where the discrete $k$ are assumed to be close enough together to be reasonably described by electron bands.

\section{A. The density operator $\hat{\rho}$}

Statistically, the band states are populated according to a Fermi-Dirac distribution for the given temperature, when the system is in equilibrium. The density operator $\hat{\rho}$ is a way to introduce this population into the QM problem and provide for mixed states. Of course, once the optical field is turned on, a new equilibrium can be established and the density operator can change. Its basic definition for an equilibrium situation, in terms of 
the state probabilities $w_{i}$ is

$$
\hat{\rho}_{0}=\sum_{i} w_{i}\left|\psi_{i}\right\rangle\left\langle\psi_{i}\right|
$$

For the equilibrium distribution, the weights are taken as proportional to the Fermi-Dirac occupation numbers,

$$
w_{i}=\frac{1}{N} f_{0}\left(E_{i}\right), \quad f_{0}\left(E_{i}\right)=\frac{1}{e^{\beta\left(E_{i}-E_{F}\right)}+1},
$$

where $\beta$ is the inverse temperature and $E_{F}$ is the Fermi level. This is a density matrix normalized to one for $N$ electrons in the system. The time derivative of a general $\hat{\rho}$ follows from the quantum Liouville equation:

$$
\frac{\partial \hat{\rho}}{\partial t}=\frac{1}{i \hbar}[\hat{H}, \hat{\rho}]
$$

To apply this, we consider the leading perturbation term in the Hamiltonian, which is the electric force from the optical field $\left(\hat{\mathbf{A}}_{1}\right)$. The optical magnetic force is ignored. Then the perturbation is described by the Hamiltonian

$$
\hat{H}_{1}=-\frac{e}{m_{o}} \hat{\mathbf{A}}_{1} \cdot\left(\hat{\mathbf{p}}-e \hat{\mathbf{A}}_{0}\right) .
$$

Now the total density operator is assumed to be a sum of the equilibrium operator plus some change caused by the perturbation:

$$
\hat{\rho}=\hat{\rho}_{0}+\hat{\rho}_{1}
$$

As the total Hamiltonian also is a sum of unperturbed and perturbation parts, we can use the fact that $\left[\hat{H}_{0}, \hat{\rho}_{0}\right]=0$, and ignore the small nonlinear term $\left[\hat{H}_{1}, \hat{\rho}_{1}\right] \approx 0$, then the equation of motion for the perturbation is

$$
i \hbar \frac{\partial \hat{\rho}_{1}}{\partial t} \approx\left[\hat{H}_{0}, \hat{\rho}_{1}\right]+\left[\hat{H}_{1}, \hat{\rho}_{0}\right]
$$

Now assume expansions of $\hat{H}_{1}$ and of $\hat{\rho}_{1}$ in the unperturbed basis states,

$$
\hat{H}_{1}=\sum_{i f}|f\rangle\left\langle f\left|\hat{H}_{1}\right| i\right\rangle\left\langle i\left|, \quad \hat{\rho}_{1}=\sum_{i f} c_{f i}\right| f\right\rangle\langle i| .
$$

The constants $c_{f i}=\left\langle f\left|\hat{\rho}_{1}\right| i\right\rangle$ are just the matrix elements of $\hat{\rho}_{1}$, in the $H_{0}$ basis states. After evaluation of the commutators, and assuming $e^{-i \omega t}$ time dependence for $\hat{\rho}_{1}$, the constants $c_{f i}$ are found, and the change in the density operator is found to be

$$
\hat{\rho}_{1}=\sum_{i f} \frac{\left(w_{i}-w_{f}\right)|f\rangle\left\langle f\left|\hat{H}_{1}\right| i\right\rangle\langle i|}{\hbar(\omega+i \gamma)+\left(E_{i}-E_{f}\right)} .
$$

A small imaginary part $\gamma$ has been added to the frequency to effect the turning on of the perturbation. This constant can be considered a phenomenological damping constant, or, it can be let to go to zero if the results without damping are of interest. This expression has been used in various problems by Adler ${ }^{21}$ and in the thesis of M. Prange. ${ }^{32} E_{i}$ and $E_{f}$ are energies of two states of the unperturbed Hamiltonian. One can think that the expression involves transitions between pairs of states. Obviously the oscillatory time behavior of the perturbation Hamiltonian must be reflected in a similar behavior in this part of the density matrix. Thus, we are interested only in the response in the density matrix at the same frequency as the perturbation.

\section{B. Thermal and volume averages}

To find the dielectric function, statistical averages of the polarization or the current density are necessary. This can be done by first defining a local quantum operator, $\hat{\rho}_{e}(\mathrm{r})$ for the one-electron charge density,

$$
\hat{\rho}_{e}(\mathrm{r})=e|\mathbf{r}\rangle\langle\mathbf{r}|,
$$

and another, $\hat{j}(\mathbf{r})$, for the one-electron current density,

$$
\hat{j}(\mathbf{r})=\frac{e}{2}\{|\mathbf{r}\rangle\langle\mathbf{r}|\hat{\mathbf{v}}+\hat{\mathbf{v}}| \mathbf{r}\rangle\langle\mathbf{r}|\} .
$$

The current density operator is defined in terms of the electron velocity,

$$
\hat{\mathbf{v}}=\frac{\vec{\pi}}{m_{o}}=\frac{1}{m_{o}}(\hat{\mathbf{p}}-e \hat{\mathbf{A}}) .
$$

The statistically averaged values of these operators are found from the trace with the density operator,

$$
\begin{aligned}
\rho_{e}(\mathrm{r}) & =\left\langle\hat{\rho}_{e}(\mathbf{r})\right\rangle=\operatorname{Tr}\left\{\hat{\rho} \hat{\rho}_{e}(\mathbf{r})\right\}, \\
\mathbf{j}(\mathrm{r}) & =\langle\hat{j}(\mathbf{r})\rangle=\operatorname{Tr}\{\hat{\rho} \hat{j}(\mathbf{r})\} .
\end{aligned}
$$

In a pure state $|\psi\rangle$, with density operator $\hat{\rho}=|\psi\rangle\langle\psi|$, these produce the usual expressions for the quantum charge and current densities at point $\mathbf{r}$,

$$
\begin{aligned}
\rho_{e}(\mathrm{r}) & =e|\psi(\mathbf{r})|^{2} \\
\mathbf{j}(\mathbf{r}) & =\operatorname{Re}\left\{\psi^{*}(\mathbf{r}) e \hat{\mathbf{v}} \psi(\mathbf{r})\right\} .
\end{aligned}
$$

We can also define the local polarization operator using the electron position,

$$
\hat{\mathbf{d}}(\mathbf{r})=e \hat{\mathbf{r}}|\mathbf{r}\rangle\langle\mathbf{r}|
$$

which is statistically averaged by the same procedure,

$$
\mathbf{d}(\mathbf{r})=\operatorname{Tr}\{\hat{\rho} \hat{\mathbf{d}}(\mathbf{r})\}
$$

At some point in the calculation the volume averages are desired, to describe $\epsilon(\omega)$ for the whole sample. These 
are obtained from the usual definition, say, for the charge density (the overbar indicates volume average) due to $N$ electrons in a volume $V$,

$$
\bar{\rho}_{e}=\frac{N}{V} \int d^{3} r \rho_{e}(\mathrm{r})=\frac{N e}{V} \sum_{i} w_{i} \int d^{3} r\left|\psi_{i}(\mathbf{r})\right|^{2}
$$

The individual electron states $\psi_{i}$ are unit normalized and the probabilities sum to one. This recovers an obvious result,

$$
\rho=N \bar{\rho}_{e}=\frac{N}{V} \operatorname{Tr}\{\hat{\rho} e\}=n e .
$$

For the current density and the electric polarization, there are similar expressions,

$$
\begin{gathered}
\mathbf{J}=N \overline{\mathbf{j}}=\frac{N}{V} \int d^{3} r \mathbf{j}(\mathbf{r})=\operatorname{Tr}\{\hat{\rho} n e \hat{\mathbf{v}}\} \\
\mathbf{P}=N \overline{\mathbf{d}}=\frac{N}{V} \int d^{3} r \mathbf{d}(\mathbf{r})=\operatorname{Tr}\{\hat{\rho} n e \hat{\mathbf{r}}\}
\end{gathered}
$$

For the most part, we use the averaging of the operator,

$$
\hat{\mathcal{P}}=n e \hat{\mathbf{r}},
$$

to determine the volume-averaged polarization response.

\section{Averaged electric polarization response}

The perturbation oscillates at frequency $\omega$ and therefore we get the response in the electric polarization $\mathbf{P}$ that oscillates at the same frequency, using only the change $\hat{\rho}_{1}$ in the density matrix,

$$
\mathbf{P}=\operatorname{Tr}\left\{\hat{\rho}_{1} \hat{\mathcal{P}}\right\}=n e \sum_{i f}\left\langle f\left|\hat{\rho}_{1}\right| i\right\rangle\langle i|\hat{\mathbf{r}}| f\rangle .
$$

This requires matrix elements of the position operator in the unperturbed basis states. Those can be obtained from the equation of motion in the unperturbed system,

$$
i \hbar \dot{\hat{r}}=i \hbar \hat{\mathbf{v}}=\left[\hat{\mathbf{r}}, \hat{H}_{0}\right]
$$

Then the needed matrix elements can be expressed using the velocity,

$$
\langle i|\hat{\mathbf{r}}| f\rangle=\frac{i \hbar}{\left(E_{f}-E_{i}\right)}\langle i|\hat{\mathbf{v}}| f\rangle
$$

The optical electric field is $\mathbf{E}=-\left(\partial \hat{\mathbf{A}}_{1} / \partial t\right)=i(\omega+$ $i \gamma) \hat{\mathbf{A}}_{1}$, including the turning on of the perturbation. The perturbation can be expressed now as

$$
\hat{H}_{1}=-e \hat{\mathbf{A}}_{1} \cdot \hat{\mathbf{v}}=\frac{-e}{i(\omega+i \gamma)} \mathbf{E} \cdot \hat{\mathbf{v}}
$$

The operator $\hat{\mathbf{v}}$ need include only the DC vector potential, $\hat{\mathbf{A}}_{0}$. Then the matrix elements of both $\hat{\rho}$ and $\hat{H}_{1}$ come from the velocity. The result for the averaged electric polarization is expressed as

$$
\mathbf{P}=\frac{n e^{2} \hbar}{(\omega+i \gamma)} \sum_{i f} \frac{\left(w_{i}-w_{f}\right)\langle i|\hat{\mathbf{v}}| f\rangle\langle f|\mathbf{E} \cdot \hat{\mathbf{v}}| i\rangle}{\left[\hbar(\omega+i \gamma)+E_{i}-E_{f}\right]\left(E_{i}-E_{f}\right)}
$$

As $\mathbf{E}$ is assumed to oscillate at frequency $\omega$, this is indeed the response oscillating at that same frequency. The damping $\gamma$ is necessary so that an appropriate limit gives the classical damped responses found earlier.

With the transition frequencies given by

$$
\hbar \omega_{i f}=E_{i}-E_{f}
$$

the susceptibility components that result from (78) are

$$
\chi_{a b}=\frac{n e^{2}}{\epsilon_{0} \hbar(\omega+i \gamma)} \sum_{i f} \frac{\left(w_{i}-w_{f}\right)\left\langle i\left|\hat{v}_{a}\right| f\right\rangle\left\langle f\left|\hat{v}_{b}\right| i\right\rangle}{\omega_{i f}\left(\omega+i \gamma+\omega_{i f}\right)} .
$$

We apply this to find only the effects from interband transitions. The free electron response in (34) is still applied for the quantum model.

The result can be symmetrized by labeling some states as occupied states (o) and the rest as unoccupied ( $\mathrm{u}$ ). All terms correspond to transitions from occupied to unoccupied states. In this way the expression becomes

$$
\chi_{a b}=\frac{n e^{2}}{\epsilon_{0} \hbar(\omega+i \gamma)} \sum_{i}^{o} \sum_{f}^{u} \frac{w_{i}-w_{f}}{\omega_{i f}}\left\{\frac{\left\langle i\left|\hat{v}_{a}\right| f\right\rangle\left\langle f\left|\hat{v}_{b}\right| i\right\rangle}{\omega+i \gamma+\omega_{i f}}+\frac{\left\langle i\left|\hat{v}_{b}\right| f\right\rangle\left\langle f\left|\hat{v}_{a}\right| i\right\rangle}{\omega+i \gamma-\omega_{i f}}\right\}
$$

\section{Application to band models}

To apply this result, we need to use the energy levels appropriate for the bands under consideration. The dis- cussion is restricted to a parabolic two-band model, with the bands separated by some gap energy $E_{g}$. There are effective masses $m_{h}^{*}$ and $m_{e}^{*}$ for the occupied (lower) and unoccupied (higher) bands, respectively. Each band is affected by the Zeeman shift in the same direction; there 
are not Landau level shifts. One can measure energies from the top of the lower band. Then the energies $E_{i}$ for the occupied band ( $E_{h}$, valence band) and the energies $E_{f}$ for the unoccupied band ( $E_{e}$, conduction band) are assumed to be

$$
\begin{aligned}
& E_{i}=E_{h}=-\frac{\hbar^{2} \mathbf{k}_{i}^{2}}{2 m_{h}^{*}}-\frac{1}{2} m_{i} \hbar \omega_{B}, \\
& E_{f}=E_{e}=E_{g}+\frac{\hbar^{2} \mathbf{k}_{f}^{2}}{2 m_{e}^{*}}-\frac{1}{2} m_{f} \hbar \omega_{B},
\end{aligned}
$$

These Zeeman shifts apply to positive charges; they are reversed in sign for negative charges, taking $\omega_{B}<0$. The azimuthal quantum numbers are $m_{i}$ and $m_{f}$. They are restricted by the orbital angular momentum numbers for each band, $l_{i}$ and $l_{f}$, respectively. Assuming vertical transitions that conserve linear momentum $\hbar \mathbf{k}$ (negligible photon momentum), the transition energies are

$$
\hbar \omega_{i f}=-E_{g}-\frac{\hbar^{2} \mathbf{k}^{2}}{2 m^{*}}-\frac{1}{2}\left(m_{i}-m_{f}\right) \hbar \omega_{B},
$$

where the reduced mass $m^{*}$ is defined by

$$
\frac{1}{m^{*}}=\frac{1}{m_{e}^{*}}+\frac{1}{m_{h}^{*}} .
$$

We write the transition frequencies in the following manner:

$$
\omega_{i f}=-\omega_{g}-s^{2}+\frac{1}{2} \Delta m \omega_{B},
$$

where the gap frequency $\omega_{g}$, scaled wave vector $s$, and change in azimuthal quantum number $\Delta m$ are

$$
\omega_{g} \equiv \frac{E_{g}}{\hbar}, \quad s \equiv \sqrt{\frac{\hbar}{2 m^{*}}} k, \quad \Delta m \equiv m_{f}-m_{i} .
$$

Only momentum-conserving transitions between two selected bands at some wave vector $\mathbf{k}$ are considered. The matrix elements needed are approximated in a form

$$
\left\langle\mathbf{k}^{\prime} l^{\prime} m^{\prime}\left|\hat{v}_{x}\right| \mathbf{k} l m\right\rangle=\frac{\hbar k_{x}}{m_{o}} M(\mathbf{k}) \delta_{\mathbf{k}^{\prime}, \mathbf{k}} \delta_{l^{\prime}, l \pm 1} \delta_{m^{\prime}, m \pm 1} .
$$

The last Kronecker deltas reflect the electric dipole selection rules, $\Delta l= \pm 1, \Delta m= \pm 1$. The dimensionless matrix element $M(\mathbf{k})$ is assumed to be some constant for the transitions of interest.

These velocity matrix elements are proportional to corresponding position matrix elements, see (76), or even the matrix elements of the $\vec{\pi}$ operator. We only need the components of operators along $x$ and $y$. But the angular part of these matrix elements is due to the electric dipole selection rules. That angular part has the following symmetries, from matrix elements between spherical harmonics,

$$
\left\langle l^{\prime} m^{\prime}\left|\hat{v}_{y}\right| l m\right\rangle=-i \Delta m\left\langle l^{\prime} m^{\prime}\left|\hat{v}_{x}\right| l m\right\rangle, \quad \Delta m= \pm 1 .
$$

This directly affects the susceptibility for each circular polarization. From (80) we have the diagonal part as

$$
\chi_{x x} \sim \sum_{f i} g_{f i}\left|\left\langle f\left|\hat{v}_{x}\right| i\right\rangle\right|^{2}
$$

but the off-diagonal part as

$$
\chi_{x y} \sim \sum_{f i}(-i \Delta m) g_{f i}\left|\left\langle f\left|\hat{v}_{x}\right| i\right\rangle\right|^{2} .
$$

It is clear that $\tilde{\chi}$ and $\tilde{\epsilon}$ have the same symmetry. Then the susceptibilities for the right and left circular polarizations vary like

$$
\begin{aligned}
& \chi_{R}=\chi_{x x}-i \chi_{x y} \sim \sum_{f i}(1-\Delta m) g_{f i}\left|\left\langle f\left|\hat{v}_{x}\right| i\right\rangle\right|^{2} \\
& \chi_{L}=\chi_{x x}+i \chi_{x y} \sim \sum_{f i}(1+\Delta m) g_{f i}\left|\left\langle f\left|\hat{v}_{x}\right| i\right\rangle\right|^{2}
\end{aligned}
$$

In these expressions, only $\Delta m=-1(\Delta m=+1)$ contributes to $\chi_{R}\left(\chi_{L}\right)$. Each factor is a Kronecker delta, i.e., $(1 \pm \Delta m)=2 \delta_{m_{f}=m_{i} \pm 1}$. The following expressions result for integration in the band model expressed using the transitions between occupied (lower band) and unoccupied (higher band) states:

$$
\begin{aligned}
& \chi_{R}=\frac{2 n e^{2}}{\epsilon_{0} \hbar(\omega+i \gamma)} \sum_{i}^{o} \sum_{f}^{u} \frac{w_{i}-w_{f}}{\omega_{i f}}\left|\left\langle f\left|\hat{v}_{x}\right| i\right\rangle\right|^{2}\left\{\frac{\delta_{m_{f}=m_{i}-1}}{\omega+i \gamma+\omega_{i f}}+\frac{\delta_{m_{f}=m_{i}+1}}{\omega+i \gamma-\omega_{i f}}\right\} \\
& \chi_{L}=\frac{2 n e^{2}}{\epsilon_{0} \hbar(\omega+i \gamma)} \sum_{i}^{o} \sum_{f}^{u} \frac{w_{i}-w_{f}}{\omega_{i f}}\left|\left\langle f\left|\hat{v}_{x}\right| i\right\rangle\right|^{2}\left\{\frac{\delta_{m_{f}=m_{i}+1}}{\omega+i \gamma+\omega_{i f}}+\frac{\delta_{m_{f}=m_{i}-1}}{\omega+i \gamma-\omega_{i f}}\right\} .
\end{aligned}
$$


The only difference between these is the swapping of the Kronecker deltas. Then the two cases can be written in terms of a single expression, replacing the \pm 1 in the Kronecker deltas with the helicity index:

$$
\begin{aligned}
\chi_{\nu}= & \frac{2 n e^{2}}{\epsilon_{0} \hbar(\omega+i \gamma)} \sum_{i}^{o} \sum_{f}^{u} \frac{w_{i}-w_{f}}{\omega_{i f}}\left|\left\langle f\left|\hat{v}_{x}\right| i\right\rangle\right|^{2} \\
& \times\left\{\frac{\delta_{m_{f}=m_{i}+\nu}}{\omega+i \gamma+\omega_{i f}}+\frac{\delta_{m_{f}=m_{i}-\nu}}{\omega+i \gamma-\omega_{i f}}\right\} .
\end{aligned}
$$

To proceed further, it is necessary to evaluate the sums. This can be facilitated by converting them to integrals over the allowed transitions, which depends slightly on the dimensionality of the bands under consideration.

\section{E. Interband transitions between three-dimensional bands}

The band structure of interest could be effectively isotropic and three-dimensional, say, for the case of some semiconductors near the $\Gamma$ point $(\mathbf{k}=0)$. Therefore it is interesting to consider the IBT contribution for this model, before doing a similar analysis of the reduced one-dimensional band model for metals.

Converting from a sum to an integral with $\sum_{\mathbf{k}} \rightarrow$ $\frac{V}{(2 \pi)^{3}} \int d \mathbf{k}$, using the assumed form for the matrix elements, and then changing to $s=\sqrt{\hbar / 2 m^{*}} k$ as the variable of integration, the interband susceptibility can be written

$$
\chi_{\nu}=Q T_{\nu}(\omega),
$$

where $Q$ contains all the constant normalization factors, and the interband transition integral $T_{\nu}(\omega)$ contains all of the frequency and temperature dependence:

$$
\begin{aligned}
Q= & \frac{2 n e^{2}|M|^{2} \hbar}{m_{o}^{2} \epsilon_{0}} \frac{V}{(2 \pi)^{3}} \frac{4 \pi}{3}\left(\frac{2 m^{*}}{\hbar}\right)^{5 / 2}, \\
T_{\nu}= & \frac{1}{\omega+i \gamma} \sum_{m_{i}} \sum_{m_{f}} \int_{0}^{s_{F}} d s \frac{w_{i}-w_{f}}{\omega_{i f}} s^{4} \\
& \times\left\{\frac{\delta_{m_{f}=m_{i}+\nu}}{\omega+i \gamma+\omega_{i f}}+\frac{\delta_{m_{f}=m_{i}-\nu}}{\omega+i \gamma-\omega_{i f}}\right\},
\end{aligned}
$$

To arrive at this, $\int d \Omega k_{x}^{2}=\frac{4 \pi}{3} k^{2}$ was used for the angular part of the integration. The upper limit is a Fermi wave vector $s_{F}=\sqrt{\hbar / 2 m^{*}} k_{F}$ needed to sum over all the states of the occupied initial band. We can let $w_{i}=1$ for the lower band, but keep the the temperature-dependent occupation probability $w_{f}>0$ for the upper band. In this way, any thermal effects due to an initial population in the final band will be included.

The first integral in (100) uses $\Delta m=+\nu$ and the second uses $\Delta m=-\nu$. These choices enter in the expression for $\omega_{i f}(\Delta m)$. In terms of the scaled wave vector $s$ or a related excitation variable $x=\omega_{g}+s^{2}$, one has

$$
\begin{aligned}
& \omega_{i f}(+\nu)=-\omega_{g}-s^{2}+\nu \frac{\omega_{B}}{2}=-x+\zeta_{\nu}, \\
& \omega_{i f}(-\nu)=-\omega_{g}-s^{2}-\nu \frac{\omega_{B}}{2}=-x-\zeta_{\nu} .
\end{aligned}
$$

The variable $x$ is the excitation energy above the lower band, and $\zeta_{\nu}=\frac{1}{2} \nu \omega_{B}$ is the polarization-dependent Zeeman splitting. Then the denominators in (100) in the two terms are $\left(\omega+i \gamma+\zeta_{\nu}-x\right)$ and $\left(\omega+i \gamma+\zeta_{\nu}+x\right)$, respectively. This suggests introducing notation for a Zeeman-shifted complex optical frequency, for the two circular polarizations:

$$
\omega_{\nu} \equiv \omega+i \gamma+\zeta_{\nu}=\omega+i \gamma+\frac{1}{2} \nu \omega_{B} .
$$

Thus, most of the polarization-dependent effects will be carried by this shifted frequency.

To include the Fermi occupation factor $w_{i}-w_{f}$, the sum over $m_{i}$ in (100) can be done first, holding $m_{f}$ fixed. Then both terms will have the same occupation factor, taken to be $1-w_{f}$. The first term in (100) uses only $m_{i}=$ $m_{f}-\nu$, the second uses only $m_{i}=m_{f}+\nu$, assuming those states exist in the lower band. The final state energy, however, depends on the effective mass $m_{e}^{*}$ in the upper band, whereas the transition energy depends on the reduced mass $m^{*}$. If these are nearly the same, i.e., $m_{e}^{*} \approx m_{h}^{*} \approx m^{*}$, then the final state energy measured relative to the top of the lower band can be taken as

$$
E_{f} \approx \hbar\left(\omega_{g}+s^{2}-\frac{1}{2} m_{f} \omega_{B}\right)=\hbar\left(x-\frac{1}{2} m_{f} \omega_{B}\right) .
$$

This will lead to an occupation factor $w_{i}-w_{f}$ for each term determined from the Fermi energy $E_{F}$,

$$
g_{m_{f}}(x) \approx 1-F\left(E_{f}-E_{F}\right)=1-F\left(x ; m_{f}\right) .
$$

Inouye et al. ${ }^{14}$ and also Scaffardi and Tocho ${ }^{15}$ have discussed bound electron response in a 1D band model without $\mathbf{B}$, applying an expression which is an integral over the excitation variable $x$. We can transform the integral $I_{\nu}$ into an integration over $x$ to compare with those results. In the simplest case, when the lower band has a higher angular momentum $l_{i}$ than the upper band value, $l_{f}$, then both states $m_{i}=m_{f} \pm \nu$ are available for all the allowed $m_{f}$. In this case, Eq. (100) becomes

$$
T_{\nu}=\frac{\omega_{2 \nu}}{\omega+i \gamma} \sum_{m_{f}} \int_{\omega_{g}}^{x_{F}} d x \frac{g_{m_{f}}(x) x\left(x-\omega_{g}\right)^{3 / 2}}{\left(x^{2}-\frac{1}{4} \omega_{B}^{2}\right)\left(x^{2}-\omega_{\nu}^{2}\right)} .
$$

The function $g_{m_{f}}=w_{i}-w_{f}$ includes the magnetic field effects on the final state occupation probabilities, which are slightly different for each $m_{f}$ level. The upper limit is determined by the scaled wave vector at the Fermi level, $x_{F}=\omega_{g}+s_{F}^{2}$. Although this is the a 3D band model, the similarity to the corrected expression from Scaffardi and Tocho ${ }^{15}$ is clear. When reduced to one dimension (use 
$\sqrt{x-\omega_{g}}$ in place of $\left(x-\omega_{g}\right)^{3 / 2}$, together with a different constant factor $Q$ out front), it recovers the expression from Ref. 15 for $\omega_{B} \rightarrow 0$.

The main effect of the DC magnetic field is to shift the optical frequency oppositely for the two circular polarizations. The second important effect is the modification of the $m_{f}$ occupations with $B$. There appears another effect due to the factor $x^{2}-\frac{1}{4} \omega_{B}^{2}$ in the denominator, however, that is relatively small, quadratic in the field, and not dependent on the polarization. The dependence on $2 \nu$ in the numerator is interesting.

In the limit where the initial states are fully occupied and the final states are fully unoccupied (all $w_{f}=0$ ), these integrals can be carried out analytically, see Appendix A. In the further approximation where the upper limit of integration is set to $s_{F} \rightarrow \infty$, the result for this complex integral is:

$$
\begin{aligned}
T_{\nu}= & \frac{\pi\left(l_{i}+l_{f}\right)}{2(\omega+i \gamma)^{2}}\left\{i\left(\omega_{\nu}-\omega_{g}\right)^{3 / 2}+\left(\omega_{\nu}+\omega_{g}\right)^{3 / 2}\right. \\
& \left.-\left(\omega_{g}-\zeta_{\nu}\right)^{3 / 2}-\left(\omega_{g}+\zeta_{\nu}\right)^{3 / 2}\right\}
\end{aligned}
$$

The lower and upper band angular momenta combine to give the multiplicity of transitions, $g_{m}=l_{i}+l_{f}$. This result corresponds to the situation of intrinsic semiconductor particles where the Fermi level is near or below the middle of the gap. This limit takes out any temperature dependence. However, that should be a small effect only from the rounding of the occupation of levels near the Fermi energy. Assuming that the gap frequency $\omega_{g}$ is large compared to the Zeeman splitting $\omega_{B}$, temperature effects on the FR could be small. Furthermore, the limit of zero damping is simple to read out from this expression. However, generally, we can calculate all results more precisely using the full theory result in equation (106).

\section{F. A 1D band model}

The band structure in real materials can be very complicated. The isotropic 3D model just discussed is an idealization for real solids. In the band structure of gold $^{16}$ in the $\mathrm{L}$ direction at the Fermi surface, the important IBTs occur from the $\mathrm{d}$ valence band to the spconduction band, where the two bands are separated by a gap of $E_{g} \approx 2 \mathrm{eV}$. Because this happens along a particular direction, a 1D band model is useful. Inouye et al. ${ }^{14}$ used a 1D band model to get the absorption contribution in gold due to the interband transitions for bound electrons. They were able to fit the absorption very well for photon energies above $2 \mathrm{eV}$ up into the ultraviolet, well above the small particle plasmon resonance frequency. Without interband transitions taken into account, it is impossible to get such an accurate description.
The main difference from the $3 \mathrm{D}$ problem, is that the integration may start out in $3 \mathrm{D}$, but needs to be reduced to an effective integration along only the active direction of the band, which is taken as the $k_{x}$-axis. The details of how this is done are not so important. One way is to convert the sum in (97) into integration in a box along Cartesian axes, using that to define an effective Fermi wave vector (slightly different from the standard definition) by

$$
N=\frac{2 V}{(2 \pi)^{3}} \int_{-k_{F}}^{k_{F}} \int_{-k_{F}}^{k_{F}} \int_{-k_{F}}^{k_{F}} d k_{x} d k_{y} d k_{z}=\frac{2 V k_{F}^{3}}{\pi^{3}} .
$$

Then an effective Fermi wave vector is defined here as $k_{F}=\pi(n / 2)^{1 / 3}$, which differs from the usual expression $k_{F}=\left(3 \pi^{2} n\right)^{1 / 3}$ by the factor $(\pi / 6)^{1 / 3} \approx 0.8$, of no real importance. Now in the sum over wave vectors in (97), the transverse (inactive) coordinates $y, z$ are integrated out, leaving only the integration over the band coordinate:

$$
\sum_{\mathbf{k}_{i}} \rightarrow \frac{2 V}{(2 \pi)^{3}}\left(2 k_{F}\right)^{2} \int_{-k_{F}}^{k_{F}} d k_{x}=\frac{V k_{F}^{2}}{\pi^{3}} \int_{-k_{F}}^{k_{F}} d k_{x}
$$

Normalized this way, this final integration will correctly give a dimensionless result for $\chi_{\nu}$. The conversion from sum to integration is carried out by including the factor $V k_{F}^{2} / \pi^{3}$ rather than the more usual factor $V /(2 \pi)^{3}$ used for $3 \mathrm{D}$. Then for $1 \mathrm{D}$, the interband susceptibility can also be written $\chi_{\nu}=Q T_{\nu}(\omega)$, the latter being an integration over $s=\sqrt{\hbar / 2 m^{*}} k_{x}$, and containing the frequency and temperature dependence,

$$
\begin{aligned}
Q= & \frac{2 n e^{2}|M|^{2} \hbar}{m_{o}^{2} \epsilon_{0}} \frac{2 V k_{F}^{2}}{\pi^{3}}\left(\frac{2 \tilde{m}}{\hbar}\right)^{3 / 2} \\
T_{\nu}= & \frac{1}{\omega+i \gamma} \sum_{m_{i}} \sum_{m_{f}} \int_{0}^{s_{F}} d s \frac{w_{i}-w_{f}}{\omega_{i f}} s^{2} \\
& \times\left\{\frac{\delta_{m_{f}=m_{i}+\nu}}{\omega+i \gamma+\omega_{i f}}+\frac{\delta_{m_{f}=m_{i}-\nu}}{\omega+i \gamma-\omega_{i f}}\right\}
\end{aligned}
$$

This is very similar to the expression (100) for $3 \mathrm{D}$. The only difference is the factor $s^{2}$ for $1 \mathrm{D}$ in place of $s^{4}$ in $3 \mathrm{D}$.

The expression for $T_{\nu}$ can be written with the excitation variable $x=\omega_{g}+s^{2}$; In the most general case, both states $m_{i}=m_{f} \pm \nu$ do not exist for all choices of $m_{f}$, and the two terms in the integrand cannot be combined in a simple form. One must then evaluate (111) by

$$
\begin{aligned}
T_{\nu}= & \frac{-1}{\omega+i \gamma} \sum_{m_{f}} \int_{\omega_{g}}^{x_{F}} d x g_{m_{f}}(x) \frac{\sqrt{x-\omega_{g}}}{x-\zeta_{\nu}} \times \\
& \times \frac{1}{2} \sum_{m_{i}}\left\{\frac{\delta_{m_{i}=m_{f}-\nu}}{\omega_{\nu}-x}+\frac{\delta_{m_{i}=m_{f}+\nu}}{\omega_{\nu}+x}\right\}
\end{aligned}
$$


When the angular momentum $l_{f}$ of the upper band is less than that of the lower band, $l_{i}$, both the choices $m_{i}=m_{f} \pm \nu$ always exist for any $m_{f}$, and this can be written instead as

$$
T_{\nu}=\frac{\omega_{2 \nu}}{\omega+i \gamma} \sum_{m_{f}} \int_{\omega_{g}}^{x_{F}} d x \frac{g_{m_{f}}(x) x \sqrt{x-\omega_{g}}}{\left(x^{2}-\frac{1}{4} \omega_{B}^{2}\right)\left(x^{2}-\omega_{\nu}^{2}\right)} .
$$

As mentioned earlier, the only change from the $3 \mathrm{D}$ expression is that the power is now $\frac{1}{2}$ instead of $\frac{3}{2}$ in the numerator. That is a complex integrand. If the real and imaginary parts are needed, it can be written in terms of real quantities, better for comparison with the $B=0$ equations:

$$
\begin{aligned}
T_{\nu}= & \frac{\omega_{2 \nu}}{\omega+i \gamma} \sum_{m_{f}} \int_{\omega_{g}}^{x_{F}} d x g_{m_{f}}(x) \frac{x \sqrt{x-\omega_{g}}}{x^{2}-\frac{1}{4} \omega_{B}^{2}} \\
& \times \frac{\left(x^{2}+\gamma^{2}-\omega_{F}^{2}\right)+2 i \gamma \omega_{F}}{\left(x^{2}+\gamma^{2}-\omega_{F}^{2}\right)^{2}+4 \gamma^{2} \omega_{F}^{2}} .
\end{aligned}
$$

Here a notation for a real shifted Faraday frequency is used,

$$
\omega_{F} \equiv \omega+\frac{1}{2} \nu \omega_{B},
$$

that is dependent on the polarization index $\nu$. In the application of this to gold nanoparticles, we assume transitions from the lower d-band to the upper p-band, hence there are values $m_{f}=-1,0,1$ to be summed over.

The last result also has an approximate expression that applies if the Fermi energy falls well within the band gap. Then, the Fermi occupation factors can be approximated with $w_{i}-w_{f}=1$, removing all the temperature dependence. The integral for $T_{\nu}$ can be done exactly in this case, see the Appendix. There results

$$
\begin{aligned}
T_{\nu}= & \frac{\pi\left(l_{i}+l_{f}\right)}{2(\omega+i \gamma)^{2}}\left\{i \sqrt{\omega_{\nu}-\omega_{g}}-\sqrt{\omega_{\nu}+\omega_{g}}\right. \\
& \left.+\sqrt{\omega_{g}-\zeta_{\nu}}+\sqrt{\omega_{g}+\zeta_{\nu}}\right\} .
\end{aligned}
$$

Obviously the expression is similar to that for the 3D band model, but now the powers are $\frac{1}{2}$ instead of $\frac{3}{2}$. A comparison of the full integral (114) and this approximation (116), as functions of the photon energy, can be seen in the Appendix in Figure 7.

\section{G. Interband parameters for gold}

Following Inouye et al..$^{14}$ and also Scaffardi and Tocho ${ }^{15}$, the 1D band model was applied here for the dielectric response of gold nanoparticles. As for the Drude model, we can fit the 1D interband permittivity, Eq. (114) for $B=0$ to the absorption of a solution of 17 $\mathrm{nm}$ diameter gold particles in water. The absorption

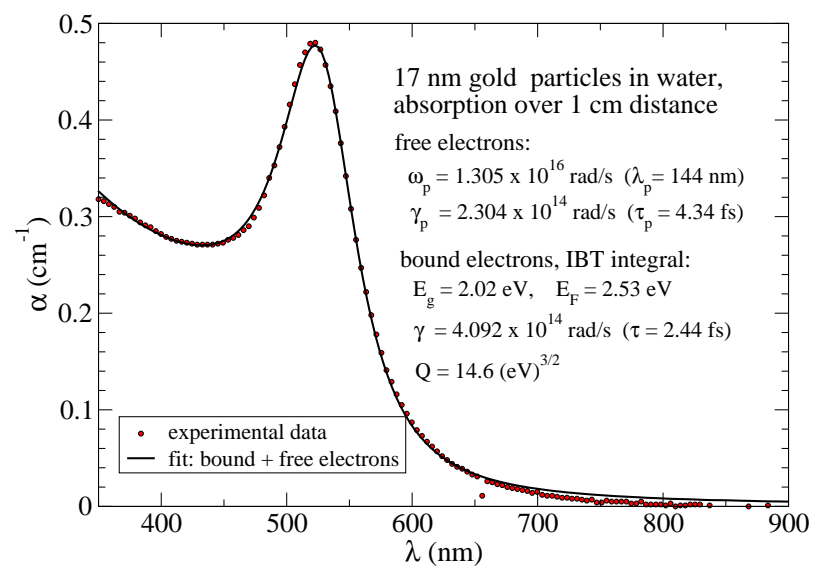

FIG. 4: (Color online) Fitting of the absorption of $17 \mathrm{~nm}$ diameter gold particles in water solution, according to the 1D band model for the interband dielectric response. Fit was made via a Monte Carlo search, allowing both the bound electron and free electron parameters to be varied. Their final adjusted values, for $T=300 \mathrm{~K}$, are indicated on the Figure. The fitted gold volume fraction is $f_{s}=5.95 \times 10^{-7}$.

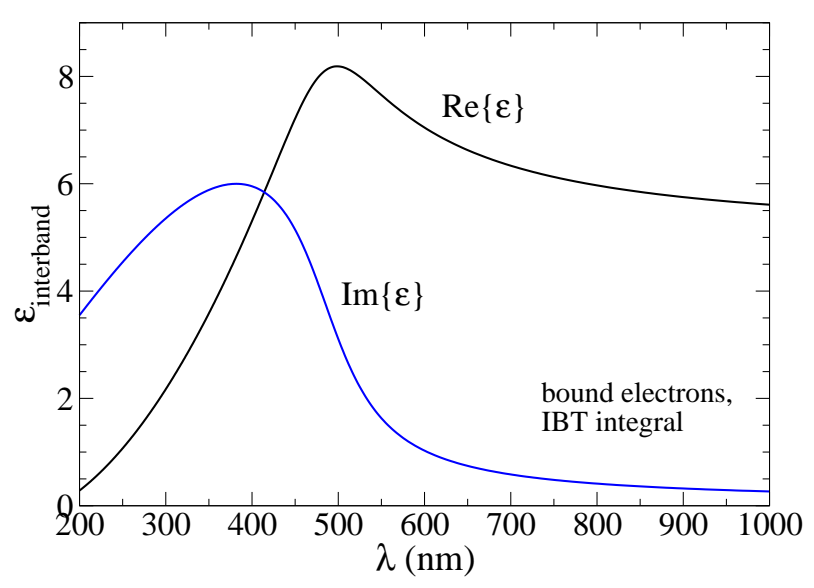

FIG. 5: (Color online) The IBT contribution to the permittivity, from the 1D band model, Eq. (114), using the parameters of Figure 4. In this model both the real and imaginary parts remain positive for all frequencies.

data from $350 \mathrm{~nm}$ - $900 \mathrm{~nm}$ were fit to Eq. (114) (using also the MG theory presented earlier) while allowing the gap energy $\hbar \omega_{g}$, Fermi energy $E_{F}$, bound electron damping $\gamma$, and normalization constant $Q$ to be varied. We also allow the gold volume fraction $f_{s}$ and free electron plasma frequency $\omega_{p}$ and damping $\gamma_{p}$ to be varied.

This is a multi-parameter search, which was carried out via a Metropolis Monte Carlo algorithm. For the effective energy function to be minimized, it was found practical to use the sum of the absolute differences between the experimental data $\alpha_{i}$ at each frequency and 
the theoretical expression $\alpha_{\mathrm{th}}$, i.e., $\sum_{i}\left|\alpha_{i}\left(\omega_{i}\right)-\alpha_{\mathrm{th}}\left(\omega_{i}\right)\right|$, instead of the squares (this gives a more uniform weighting but looser fit to the points). A reasonable fit and the associated parameters are shown in Figure 4. The gap energy $E_{g}=2.02 \mathrm{eV}$, Fermi energy $E_{F}=2.53 \mathrm{eV}$ and plasma frequency $\left(\hbar \omega_{p}=8.59 \mathrm{eV}\right)$ determined in this fit are consistent with the values used in Ref. 15 . The dampings, $\hbar \gamma_{p}=0.152 \mathrm{eV}\left(\gamma_{p}^{-1}=4.33 \mathrm{fs}\right)$ and $\hbar \gamma=0.269 \mathrm{eV}\left(\gamma^{-1}=2.45 \mathrm{fs}\right)$, are somewhat different from those for bulk gold, and the resulting real and imaginary parts of dielectric function $\epsilon(\omega)$ are different from those for bulk gold. ${ }^{33}$ However, $\gamma_{p}$ is consistent with the prediction from Eq. (43), as expected due to extra surface scattering and other factors for the nanoscale particles. If this surface scattering effect is taken out, the model reproduces the real and imaginary parts of $\epsilon(\omega)$ for bulk gold that are completely consistent with those found by Johnson and Christy. ${ }^{33}$

A value of gold volume fraction $f_{s}=5.95 \times 10^{-7}$ was needed in the fit. It is about half the value estimated by the techniques in Ref. 26, see Sec. II, showing the difficulty in estimation of $f_{s}$ in the lab. We might note that this fit is not strongly constrained; it was determined only by the absorption data; other values cannot be strongly ruled out. Unlike the Drude model presented earlier, this model is very good at fitting the ultraviolet end of the dielectric properties, and also fits the infrared end better. Thus, we expect it should give more reliable predictions for the Faraday rotation properties.

\section{FARADAY ROTATION DUE TO GOLD NANOPARTICLES}

Here we apply the 1D band model to the interband transitions, and compare the theory to experiments for gold NPs. Based on the fitting of parameters for 17 $\mathrm{nm}$ diameter gold particles in the previous section, the results for scaled complex Faraday rotation angle $\Psi=$ $\Upsilon+i Z=(\varphi+i \mathcal{X}) /\left(B z f_{s}\right)$ can be estimated using Eq. (15) together with the MG theory for the response of a dilute solution, Eq. (40). The real and imaginary parts are the Verdet and ellipticity factors, respectively, scaled by volume fraction $f_{s}$ of gold in the solution.

The theory results for $17 \mathrm{~nm}$ diameter gold NPs are compared with experimental data for the FR spectrum in Fig. 6. The experimental data for $\Upsilon=v / f_{s}$ has been scaled down by a factor of $1 / 10$ so that its negative FR peak is similar in magnitude to the theory. Although some details are not identical between theory and experiment, the general trend in $\Upsilon$ as a function of wavelength is similar. Both show a strong negative peak due to the plasmon near $520-530 \mathrm{~nm}$, and a zero crossing in $\Upsilon$ near $550 \mathrm{~nm}$. This peak is about ten times stronger when using the IBT theory than the simpler Drude approach presented in Fig. 3, but the negative

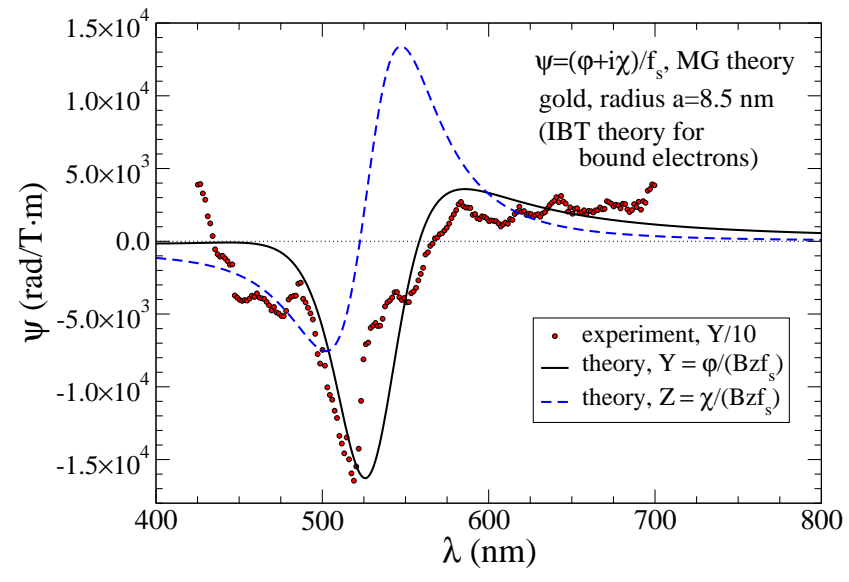

FIG. 6: (Color online) (a) The real and imaginary parts of the scaled complex Faraday rotation $\Psi=(\varphi+i \mathcal{X}) /\left(B z f_{s}\right)$, from experiment (points), and calculated including IBTs for bound electrons in the 1D band model for gold particles of 17 nm diameter (lines). The real part (solid curve) is the Verdet factor per volume fraction, $\Upsilon$. The experimental data for $\Upsilon$ has been scaled by a factor of $1 / 10$, which brings its peak to the same size as in the IBT theory. The imaginary part is the ellipticity factor scaled by volume fraction (not measured).

FR peak in the experimental data is still much stronger than in both theories. The experimental result for $\Upsilon$, however, is obtained by dividing raw FR data by the estimated gold volume fraction (using techniques of Ref. $26), f_{s}=1.23 \times 10^{-6}$. Any error in the volume fraction will modify the experimental value of $\Upsilon$. The volume fraction divides out in the theory for $\Upsilon$. Various multiple scattering and backscattering effects ${ }^{11}$ and other similar aggregation effects ${ }^{10}$ not included in this theory could explain this large discrepancy.

Above $560 \mathrm{~nm}$ both theory and experiment indicate a positive Faraday rotation angle. According to the theory, there is also a strong positive peak in ellipticity expected around a wavelength slightly larger than that due to the plasmon (around $550 \mathrm{~nm}$ ). Notably, both the rotation and ellipticity tend towards zero at short wavelengths, removing the artifact present in the classical Drude approach.

\section{DISCUSSION AND CONCLUSIONS}

The interband electronic transitions are known to have a considerable effect on dielectric properties of gold and other metals. Although it is popular to consider only a simple Drude model for quasi-free electrons, it is shown here to be inadequate for describing, say, the absorption in gold nanoparticles. A fit for the dielectric parameters based on a Drude model, combining free electron 
and bound electron contributions, was given in Fig. 1, for a dilute solution of gold NPs. The absorption peak near $520 \mathrm{~nm}$, associated with the excitation of a plasmon resonance in the NPs, can be fit rather well. In the violet and ultraviolet, however, it is impossible to get a good match between the theory and experimental data for gold particles of average diameter of $17 \mathrm{~nm}$. This approach leads (incorrectly) to negative values of the real part of permittivity at shorter wavelengths (below the plasmon resonance), which is why the fit fails in that wavelength region.

For the optical effects such as absorption or Faraday rotation, it is clear that a correct description of $\epsilon(\omega)$ for a macroscopic sample completely determines the outcome. If the absorption cannot be fitted properly, then it is hard to see how the Drude approach could explain Faraday rotation very well. Instead, we include the interband transitions for the bound electrons, based on the quantum formulation due to Boswarva et al. ${ }^{20}$ and also Adler. ${ }^{21}$. However, the calculation done here is specifically for nanoparticles in the presence of a DC magnetic field $B$. Bulk calculations ${ }^{20,22}$ have assumed Landau levels due to $B$ for an infinite sample, but the Landau radius $r_{0}=\sqrt{\frac{2 \hbar}{e B}}$ for weak $B$ will be larger than the typical NP size. Hence, the Landau levels have no physical sense for weak magnetic field; they are not the quantum energy eigenstates. The states are modified due to the geometric confinement of a limited size nanoparticle. The shift in energy states used here is a Zeeman shift due to the DC magnetic field for electrons in bands of specified angular momentum.

We are applying this calculation at a magnetic field strength such that the Landau radius is only about twice the NP radius. This more intermediate field is needed to insure adequate signal to noise ratio in the FR data. This should be within the range of the quantum theory. However, at a magnetic field strength about four times larger, the Landau radius will match the NP radius, and crossover to a more bulk-like behavior could be expected.

We have sketched out the details of the IBT integral, especially for the 1D band model. While it was required to fit the amplitude factor $Q$ and other dielectric parameters, this results in a much better fit between theory and experiment for the absorption, Fig. 4. The fitting leads to an interband permittivity whose real and imaginary parts remain positive for $\lambda>200 \mathrm{~nm}$, Fig. 5. Thus we expect this approach to be good when applied to the Faraday rotation properties of a dilute solution of NPs.

When applied to a solution of $17 \mathrm{~nm}$ diameter gold NPs, one finds a strong negative peak in the Verdet factor, as expected, near the plasmon wavelength of about $525 \mathrm{~nm}$. This negative peak is certainly due to extra Faraday rotation that is associated with the surface plasmon mode in nanoparticles. This is at least partially confirmed by experiments at a small volume fraction of gold. The theory also predicts a positive peak in ellip- ticity at a wavelength somewhat larger than the plasmon wavelength (nearly $550 \mathrm{~nm}$ for $17 \mathrm{~nm}$ gold NPs). However, at present, the experimental results for scaled Verdet factor $\Upsilon=v / f_{s}$ seem to be about an order of magnitude stronger than that indicated by this theory. Some of this discrepancy could be due to uncertainty in the true value of gold volume fraction. There may also be other processes present in the NP data, such as aggregation ${ }^{10}$ and backscattering effects, ${ }^{11}$ that are not included in the model. At present we do not have comparable experimental results on the ellipticity factor. It will be important to clarify this discrepancy in the size of the magneto-optical responses.

\section{Acknowledgments}

GMW is grateful for the hospitality of Universidade Federal de Viçosa in Viçosa, Minas Gerais, Brazil, and Universidade Federal de Santa Catarina in Florianópolis, Brazil, where parts of this work were carried out, and financial support of FAPEMIG grant BPV-00046-11. We acknowledge the support of NSF through grants NSF930673 and NSF-1128570, and the Terry Johnson Cancer Center at KSU for funding the construction of the pulsed magnet.

\section{APPENDIX A: EVALUATION OF INTEGRALS OVER SCALED WAVE VECTOR}

This analysis applies when the Fermi level is well within the band gap, and then the upper band is assumed to be unoccupied. This removes the temperature dependence from the model.

\section{3D band model}

The integral for the 3D band model, equation (100), is best evaluated using $s=\sqrt{\hbar / 2 m^{*}} k$ as the variable of integration. We assume $w_{i}=1, w_{f}=0$, and initially include the damping parameter $\gamma$. For the sum over $m_{f}$, the Kronecker deltas select $m_{f}=m_{i} \pm \nu$ for each integrand. For the sum over $m_{i}$, between bands with defined orbital angular momentum $l_{i}$ and $l_{f}$, there are multiple equivalent transitions, all with the same thermodynamic weight. This leads to a multiplicity

$$
g_{m}=\sum_{m_{i}} 1=\min \left(2 l_{i}+1,2 l_{f}+1\right)=l_{i}+l_{f} .
$$

Essentially, this constant factor replaces the temperature dependence that would have been included by the occupation function, $g_{m_{f}}(x)$ in the full theory expression. Then in this approximation, the interband susceptibility 
is $\chi_{\nu}=Q T_{\nu}(\omega)$, with $Q$ defined earlier in (99), and the transition integral expressed

$$
T_{\nu}=\frac{g_{m}}{\omega+i \gamma}\left(K_{1}+K_{2}\right) .
$$

$T_{\nu}$ has been split into two similar integrals,

$$
\begin{aligned}
& K_{1}=\int_{0}^{s_{F}} \frac{+d s s^{4}}{\left(s^{2}+\omega_{g}-\zeta_{\nu}\right)\left(s^{2}+\omega_{g}-\omega_{\nu}\right)}, \\
& K_{2}=\int_{0}^{s_{F}} \frac{-d s s^{4}}{\left(s^{2}+\omega_{g}+\zeta_{\nu}\right)\left(s^{2}+\omega_{g}+\omega_{\nu}\right)} .
\end{aligned}
$$

The shifted frequency $\omega_{\nu}$ is defined in (103) and $\zeta_{\nu}=$ $\frac{1}{2} \nu \omega_{B}$. Consider $K_{1}$ using the partial fraction expansion,

$$
K_{1}=\int_{0}^{s_{F}} \frac{d s s^{4}}{\omega_{\nu}-\zeta_{\nu}}\left[\frac{1}{s^{2}+\omega_{g}-\omega_{\nu}}-\frac{1}{s^{2}+\omega_{g}-\zeta_{\nu}}\right] .
$$

The integral $K_{2}$ is the negative of this with $\zeta_{\nu}$ and $\omega_{\nu}$ reversed in sign:

$$
K_{2}=\int_{0}^{s_{F}} \frac{d s s^{4}}{\omega_{\nu}-\zeta_{\nu}}\left[\frac{1}{s^{2}+\omega_{g}+\omega_{\nu}}-\frac{1}{s^{2}+\omega_{g}+\zeta_{\nu}}\right] .
$$

Note that $\omega_{\nu}-\zeta_{\nu}=\omega+i \gamma$. These integrals can all be found from the indefinite integral,

$$
f(s)=\int \frac{d s s^{4}}{s^{2}+a^{2}}=\frac{1}{3} s^{3}-a^{2} s+a^{3} \tan ^{-1}\left(\frac{s}{a}\right),
$$

where the parameter $a$ is complex for the four different cases where this is used. Thus the function $f(s)$ is defined with the analytic continuation of the inverse tangent to complex arguments. Applying this gives

$$
\begin{aligned}
K_{1} & =\frac{1}{\omega+i \gamma}\left\{\left(\omega_{g}-\omega_{\nu}\right)^{3 / 2} \tan ^{-1}\left(\frac{s}{\sqrt{\omega_{g}-\omega_{\nu}}}\right)\right. \\
& \left.-\left(\omega_{g}-\zeta_{\nu}\right)^{3 / 2} \tan ^{-1}\left(\frac{s}{\sqrt{\omega_{g}-\zeta_{\nu}}}\right)\right\}+s,( \\
K_{2} & =\frac{1}{\omega+i \gamma}\left\{\left(\omega_{g}+\omega_{\nu}\right)^{3 / 2} \tan ^{-1}\left(\frac{s}{\sqrt{\omega_{g}+\omega_{\nu}}}\right)\right. \\
& \left.-\left(\omega_{g}+\zeta_{\nu}\right)^{3 / 2} \tan ^{-1}\left(\frac{s}{\sqrt{\omega_{g}+\zeta_{\nu}}}\right)\right\}-s .
\end{aligned}
$$

It is interesting that once these are summed to produce $T_{\nu}=\frac{g_{m}}{\omega+i \gamma}\left(K_{1}+K_{2}\right)$, all linear and cubic terms in $s$ cancel out, leaving only the inverse tangents. These are evaluated at the upper limit $s_{F}$. Due to these cancellations, the upper limit can be let to go to infinity as a reasonable approximation (also, the choice of a finite $s_{F}$ may be difficult in any case). With $s$ real, the limit of the complex inverse tangent can be shown to have the following dependence on the parameter $a$ :

$$
\begin{aligned}
& \lim _{s \rightarrow \infty} \tan ^{-1} \frac{s}{a}=\operatorname{sgn}(\operatorname{Re}\{a\}) \frac{\pi}{2}, \\
& \lim _{s \rightarrow \infty} \tan ^{-1} \frac{i s}{a}=\operatorname{sgn}(\operatorname{Im}\{a\}) \frac{\pi}{2} .
\end{aligned}
$$

The latter form is useful for the first $\tan ^{-1}$ in $K_{1}$, changing $\sqrt{\omega_{g}-\omega_{\nu}}$ to $i \sqrt{\omega_{\nu}-\omega_{g}}$, which is more convenient when assuming above the gap excitation. Then the resulting interband integral for the 3D band model under these approximations is

$$
\begin{aligned}
T_{\nu}= & \frac{\pi g_{m}}{2(\omega+i \gamma)^{2}}\left\{i\left(\omega_{\nu}-\omega_{g}\right)^{3 / 2}+\left(\omega_{\nu}+\omega_{g}\right)^{3 / 2}\right. \\
& \left.-\left(\omega_{g}-\zeta_{\nu}\right)^{3 / 2}-\left(\omega_{g}+\zeta_{\nu}\right)^{3 / 2}\right\}
\end{aligned}
$$

These complex square roots are defined by the root with the positive real part, i.e., the root in the first or fourth quadrant. It is interesting to notice that the photon frequency does not appear inside the last factors involving the Zeeman shift $\zeta_{\nu}$. Also, in the limit of zero damping $\gamma \rightarrow 0$, the very first term is the entire imaginary part. For below the gap excitation $\left(\omega_{\nu}<\omega_{g}\right)$ the imaginary part becomes zero in the absence of damping. For typical material parameters, this approximation gives results very close to the full theoretical result from Eq. (106). This is mostly due to the high dimensionality; in the 1D band model this approximation is farther from the full theory expression.

\section{1D band model}

The analysis is nearly the same, with interband susceptibility expressed via $\chi_{\nu}=Q T_{\nu}(\omega)$. One has the same expression for the multiplicity, $g_{m}=l_{i}+l_{f}$. The weighting factor $Q$ is now given in equation (110). The expression for the transition integral can still be written

$$
T_{\nu}=\frac{g_{m}}{\omega+i \gamma}\left(K_{1}+K_{2}\right),
$$

except that in this case, the parts have the power $s^{2}$ in place of $s^{4}$ in their numerators:

$$
\begin{aligned}
& K_{1}=\int_{0}^{s_{F}} \frac{+d s s^{2}}{\left(s^{2}+\omega_{g}-\zeta_{\nu}\right)\left(s^{2}+\omega_{g}-\omega_{\nu}\right)}, \\
& K_{2}=\int_{0}^{s_{F}} \frac{-d s s^{2}}{\left(s^{2}+\omega_{g}+\zeta_{\nu}\right)\left(s^{2}+\omega_{g}+\omega_{\nu}\right)} .
\end{aligned}
$$

The partial fraction expansions are

$$
K_{1}=\int_{0}^{s_{F}} \frac{d s s^{2}}{\omega_{\nu}-\zeta_{\nu}}\left[\frac{1}{s^{2}+\omega_{g}-\omega_{\nu}}-\frac{1}{s^{2}+\omega_{g}-\zeta_{\nu}}\right],
$$




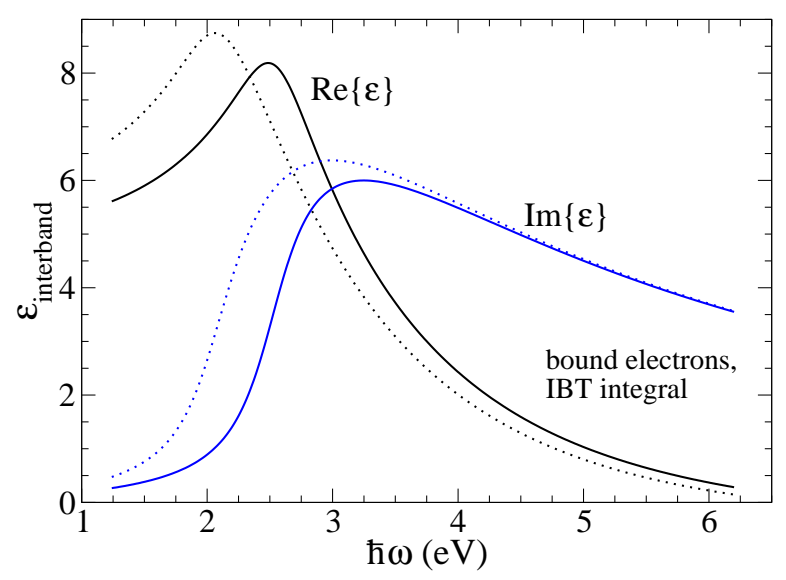

FIG. 7: (Color online) The IBT contribution to the permittivity for the 1D band model, as functions of photon energy in $\mathrm{eV}$, using the parameters of Figure 4 . The solid curves apply the full theory, Eq. (114), including the Fermi occupation factors, for temperature $300 \mathrm{~K}$. Note the peak in $\operatorname{Re}\{\epsilon\}$ at the Fermi energy $(2.52 \mathrm{eV})$ in the full theory. The dotted curves show the result of assuming a totally unoccupied upper band, $w_{f}=0$, obtaining $\epsilon(\omega)$ from Eq. (A21).

and for $K_{2}$, reverse the overall sign and the signs on $\omega_{\nu}$ and $\zeta_{\nu}$,

$$
K_{2}=\int_{0}^{s_{F}} \frac{d s s^{2}}{\omega_{\nu}-\zeta_{\nu}}\left[\frac{1}{s^{2}+\omega_{g}+\omega_{\nu}}-\frac{1}{s^{2}+\omega_{g}+\zeta_{\nu}}\right] .
$$

The basic integral needed is

$$
f(s)=\int \frac{d s s^{2}}{s^{2}+a^{2}}=s-a \tan ^{-1}\left(\frac{s}{a}\right) .
$$

Applying this to all the sub-integrals, the results are similar to those in $1 \mathrm{D}$,

$$
\begin{aligned}
K_{1} & =\frac{1}{\omega+i \gamma}\left\{-\left(\omega_{g}-\omega_{\nu}\right)^{1 / 2} \tan ^{-1}\left(\frac{s}{\sqrt{\omega_{g}-\omega_{\nu}}}\right)\right. \\
& \left.+\left(\omega_{g}-\zeta_{\nu}\right)^{1 / 2} \tan ^{-1}\left(\frac{s}{\sqrt{\omega_{g}-\zeta_{\nu}}}\right)\right\}, \quad(\mathrm{A} 19) \\
K_{2} & =\frac{1}{\omega+i \gamma}\left\{-\left(\omega_{g}+\omega_{\nu}\right)^{1 / 2} \tan ^{-1}\left(\frac{s}{\sqrt{\omega_{g}+\omega_{\nu}}}\right)\right. \\
& \left.+\left(\omega_{g}+\zeta_{\nu}\right)^{1 / 2} \tan ^{-1}\left(\frac{s}{\sqrt{\omega_{g}+\zeta_{\nu}}}\right)\right\} .
\end{aligned}
$$

Again, it will be useful to reverse the order in the radical in the first term in $K_{1}$, assuming above the gap excitation. Then we apply again, $\sqrt{\omega_{g}-\omega_{\nu}} \rightarrow i \sqrt{\omega_{\nu}-\omega_{g}}$. Letting the upper limit of integration $s_{F} \rightarrow \infty$, and inserting the limiting values of the inverse tangents, there results

$$
\begin{aligned}
T_{\nu}= & \frac{\pi g_{m}}{2(\omega+i \gamma)^{2}}\left\{i \sqrt{\omega_{\nu}-\omega_{g}}-\sqrt{\omega_{\nu}+\omega_{g}}\right. \\
& \left.+\sqrt{\omega_{g}-\zeta_{\nu}}+\sqrt{\omega_{g}+\zeta_{\nu}}\right\} .
\end{aligned}
$$

Note that for typical values of the parameters, both the real and imaginary parts of $T_{\nu}(\omega)$ are positive. This function derived has a peak in its real part, for frequency near the gap frequency $\omega_{g}$. Otherwise, it is very similar to the more complete theory of (114) that includes the varying Fermi occupation factor. That complete theory differs primarily in that the location of the peak in its real part is near the Fermi energy rather than the gap energy.
* Electronic address: wysin@phys.ksu.edu; URL: http:// www.phys.ksu.edu/personal/wysin

† Electronic address: chikan@ksu.edu

‡ Electronic address: youngnt@rose-hulman.edu; Permanent address: Rose-Hulman Institute of Technology, Terre Haute, IN 47803.

$\S$ Electronic address: rdani@ksu.edu

1 E. Hecht, Optics (4th ed.), pp. 366-368 (1997).

2 T.Y. Kim, T. Hirano, Y. Kitamoto, and Y. Yamazaki, IEEE Trans. Magn. 39, 2078 (2003).

3 Y.A. Bamakov, B.L. Scott, V. Golub, L. Kelly, V. Reddy and K.L. Stokes, J. Phys. Chem. Solids 65, 1005 (2004).

${ }^{4}$ K. Hayashi, R. Fujilkawa, W. Sakamoto, M. Inoue and T. Yogo, J. Phys. Chem. C 112, 14255 (2008).

${ }^{5}$ H.C.Y. Yu, M.A. van Eijkelenborg, S.G. Leon-Saval, A. Argyros and G.W. Barton, Appl. Opt. 47, 6497 (2008).

6 K.L. Kelly, E. Coronado, L.L. Zhao and G.C. Schatz, J. Phys. Chem. B 107, 668 (2003).

7 P. Mulvaney, Langmiur 12, 788 (1996).
8 Raj Kumar Dani, Hongwang Wang, Stefan Bossman, Gary Wysin and Viktor Chikan, Article and Supplemental Materials, J. Chem. Physics 135, 224502 (2011).

9 Prashant K. Jain, Yanhong H. Xiao, Ronald Walsworth and Adam E. Cohen, Nano Lett. 9, 1644-50 (2009).

10 Sahar Pakdel and MirFaez Miri, Phys. Rev. B 86, 235445 (2012).

11 V. Gasparian and Zh.S. Gevorkian, Phys. Rev. A 87, 053807 (2013).

$12 \mathrm{Yu} \mathrm{Gu}$ and Konstantin G. Kornev, J. Opt. Soc. Am. B 27, 2165 (2010).

13 P.M. Hui and D. Stroud, Appl. Phys. Lett. 50, 950 (1987).

14 Hideyuki Inouye, Koichiro Tanaka, Ichiro Tanahashi and Kazuyuki Hirao, Phys. Rev. B 57, 11,334 (1998).

15 Lucía B. Scaffardi and Jorge O. Tocho, Nanotechnology 17, 1309-1315 (2006).

16 N. Egede Christensen and B.O. Seraphin, Phys. Rev. B 4, 3321 (1971).

17 V. Arunasalam, J. Math. Phys. 10, 1305 (1969). 
18 L.L. Jones and H. Eyring, J. Chem. Education 38, 601 (1961).

19 N.F. Kharchenko, A.V. Bibik and V.V. Eremenko, Pis'ma Zh. Eksp. Teor. Fiz. 42, 447-9 (1985).

20 I.M. Boswarva, R.E. Howard, and A.B. Lidiard, Proc. Royal Soc. London, Series A, Mathematical and Sciences, Vol. 269, No. 1336 pp. 125-141 (1962).

21 Stephen L. Adler, Phys. Rev. 126, 413 (1962).

22 John Halpern, Benjamin Lax and Yuichiro Nishina, Phys. Rev. 134, A140 (1964).

23 J.C. Maxwell-Garnett, Philos. Trans. R. Soc. London, Ser. A 203, 385 (1904).

24 M. Born and E. Wolf, Principles of Optics, 3rd ed., Pergammon (Oxford 1965).

25 J. Turkevich, P.C. Stevenson and J. Hillier, Discussions of the Faraday Society, 11, 55 (1951).

26 Xiong Liu, Mark Atwater, Jinhai Wang and Qun Huo, Colloids and Surfaces B: Biointerfaces 58, 3 (2007).

27 T.K. Xia, P.M. Hui and D. Stroud, J. Appl. Phys. 67, 2736 (1990).

28 A positive Faraday rotation $\varphi$ corresponds to counter- clockwise $(\mathrm{CCW})$ rotation of the electric vector when the wave is approaching the observer (direction of rotation of the E-vector for left circular polarization). A positive Faraday ellipticity $\mathcal{X}$ corresponds to CCW rotation of the E-vector in its elliptical motion in time, with minor axis $b$ over major axis $a$ satisfying $\mathcal{X}=\tan ^{-1}(b / a)$. The E-vector rotates $\mathrm{CCW}$ with time, as would be the case for the left circular polarization component dominating.

29 J.D. Jackson, Electrodynamics, 5th ed., Wiley (1998).

30 Pierre Mallet, C.A. Guérin and Anne Sentenac, Phys. Rev. B 72, 014205-13 (2005).

31 Rodolphe Antoine, Pierre F. Brevet, Hubert H. Girault, Donald Bethell and David J. Schiffrin, Chem. Commun. Issue 19, p. 1901-2 (1997).

32 Micah Prange, Density matrix calculation of optical constants, PhD Thesis, University of Washington (2009), http://leonardo.phys.washington.edu/feff/papers/ dissertations/thesis_prange.pdf

33 P.B. Johnson and R.W. Christy, Phys. Rev. B 6, 4370-9 (1972). 